\title{
Analysis of airborne sound insulation and impact sound pressure level provided by a single partition containing a heterogeneity
}

\author{
A. Pereira, A. Tadeu* \\ Department of Civil Engineering, University of Coimbra, Pinhal de Marrocos, 3030-290 Coimbra, Portugal
}

Received 19 December 2005; received in revised form 7 July 2006; accepted 27 August 2006

Available online 13 November 2006

\begin{abstract}
This paper studies the acoustic behaviour provided by a single infinite elastic partition dividing an infinite acoustic medium, containing two-dimensional (2D) heterogeneities placed parallel to the layer's surfaces, which simulate the presence of fittings such as pipes. The problem is solved in the frequency domain using the boundary element method (BEM). Only the surfaces of the heterogeneities are discretized, since 2.5D Green's functions for the single layered media, bounded by acoustic media, are used. Time domain responses are also computed by applying a (fast) Fourier transform to the responses obtained in the frequency domain. The heterogeneities are assumed to be either rigid, free, fluid-filled or elastic-filled inclusions. The simulated models are used to study the contribution of the heterogeneities to the final airborne sound insulation and impact sound pressure level provided by a single partition. It was found that both airborne sound insulation and impact sound pressure level may be influenced by the presence of the heterogeneity at higher frequencies. (C) 2006 Elsevier Ltd. All rights reserved.
\end{abstract}

\section{Introduction}

In order to accurately predict the transmission of sound through a single separating partition a large number of variables needs to be considered. These variables include: the physical properties of the panel (mass, internal damping, elasticity modulus, Poisson's ratio); the finiteness of the element; the mounting conditions, and the non-diffuseness of the test room. The simulation of all the acoustic phenomena involved in the sound transmission would lead to highly complex mathematical models, and so quite a number of works found in the literature adopt simplifications to deal with the problem. Among these is the well-known theoretical Mass Law, where the element is assumed to behave like a group of infinite juxtaposed masses with independent displacement and null damping forces. Sewell [1] and Sharp [2] have proposed simplified models for the frequencies below, in the vicinity of and above the coincidence effect to calculate the airborne sound insulation of single panels, improving the prediction accuracy relative to Mass Law. More recently, Tadeu et al. [3,4] developed an algorithm for predicting the airborne sound insulation provided by a single partition based on the definitions of pressure and displacement potentials, which are combined so as to verify the

\footnotetext{
*Corresponding author. Department of Civil Engineering, Polo II of the University of Coimbra, Rua Luís Reis Santos, 3030-788 Coimbra, Portugal. Tel.: + 351239797201 ; fax: + 351239797190.

E-mail address: tadeu@dec.uc.pt (A. Tadeu).
} 
boundary conditions at the fluid/solid interfaces. Jong-Hwa et al. [5] revisit the problem of resonant transmission related to the sound insulation of rectangular finite panels in an infinite baffle at frequencies below the critical frequency by using the general modal expansion method followed by Sewell. They investigated the validity of neglecting the resonant transmission components in the prediction of transmission loss by calculating the differences between the total transmission loss and the non-resonant transmission loss. Alba et al. [6] revised the expression for an infinite impervious layer in a diffuse field and adjusted it in order to include the energy loss mechanisms (internal and coupling losses) produced in the layer. These authors further developed algorithms which can be used to obtain the mechanical properties of the layer with the appropriate set-up conditions, if databases are available.

Knowledge of the impact sound pressure level expected from partitions is also important at the design stage. Although the final responses provided by the loads that act in an acoustic or in an elastic medium differ, the dynamic behaviour of partitions may be similar. Ver [7] determined a relation between airborne sound insulation and impact sound pressure level provided by partitions. Gerretsen [8] initially proposed a model to predict airborne sound insulation including flanking transmission. His model requires knowing the direct sound reduction index provided by a partition and takes into account the boundary conditions by means of a structural reverberation time and the vibration level differences across junctions, which are calculated from in situ measurements. Since a major part of the model concerns vibration transmission and radiation, he later extended it to predict impact sound pressure level [9].

Most of the works found in the literature refer to the prediction of homogeneous partitions. However, the acoustic behaviour of real partitions may be influenced by the presence of heterogeneities, which are often present inside. These may be other building elements such as heat, sewerage, drainage or water pressure pipes or pipes that host electrical cables and wires. These are not taken into account during design, and when the performance of walls is tested the results may not match the predictions. Therefore it seems important to see if the presence of those elements can influence the sound insulation of partitions. This work aims to contribute to the analysis of this problem by developing and applying a boundary element method (BEM) model to assess the acoustic behaviour of single partitions infinite in two directions (infinite plates) dividing an infinite acoustic medium. The influence of heterogeneities in the elastic partition on the acoustic behaviour is studied by determining airborne sound insulation and impact sound pressure levels. Results for a single layer solution without discontinuities are used as a reference. The algorithm uses a Boundary Element formulation where only the heterogeneity needs to be discretized, since Green's functions for single layered media are used. These heterogeneities may be rigid, free, fluid-filled or elastic-filled inclusions. The Green's functions' solutions are the analytical solutions developed by Tadeu et al. $[3,4]$ to predict airborne sound insulation provided by a single panel. The computations are performed in the frequency domain, assuming that harmonic line loads act either in the fluid or in the elastic medium. Time domain responses are then obtained by applying an Inverse Fourier Transform. Wave propagation features occurring in a single layered medium with a heterogeneity are investigated by analysing the responses provided by different sets of receivers placed in the acoustic medium. The influence of the direction of the load on impact sound pressure level is studied. Different positions and sizes of the heterogeneity are modelled and the resulting sound level is analysed.

The next section outlines the problem formulation, indicating the Green's functions for a single layer medium, and the BEM. Then the procedure used to calculate time domain responses is summarized. The simulations are described next, and finally the results are discussed.

\section{Problem formulation}

Consider an elastic layer of thickness $h$, infinite along its plane ( $x$ and $z$ directions), dividing an infinite acoustic medium, as in Fig. 1 . The acoustic medium has a mass density $\rho_{f}$, a Lamé constant $\lambda_{f}$ and permits a dilatational wave velocity $\alpha_{f}=\sqrt{\lambda_{f} / \rho_{f}}$. The material properties of the elastic medium are the density $\rho_{s}$, Poisson's ratio $v_{\mathrm{s}}$ and a shear modulus $\mu_{s}$. In this medium the propagation occurs following compressional waves with a velocity $\alpha_{s}=\sqrt{2 \mu_{s}\left(1-v_{s}\right) / \rho_{s}\left(1-2 v_{s}\right)}$ and shear waves with a velocity $\beta_{s}=\sqrt{\mu_{s} / \rho_{s}}$. The internal material losses are considered by using a complex shear modulus and a complex Lamé constant. The shear 


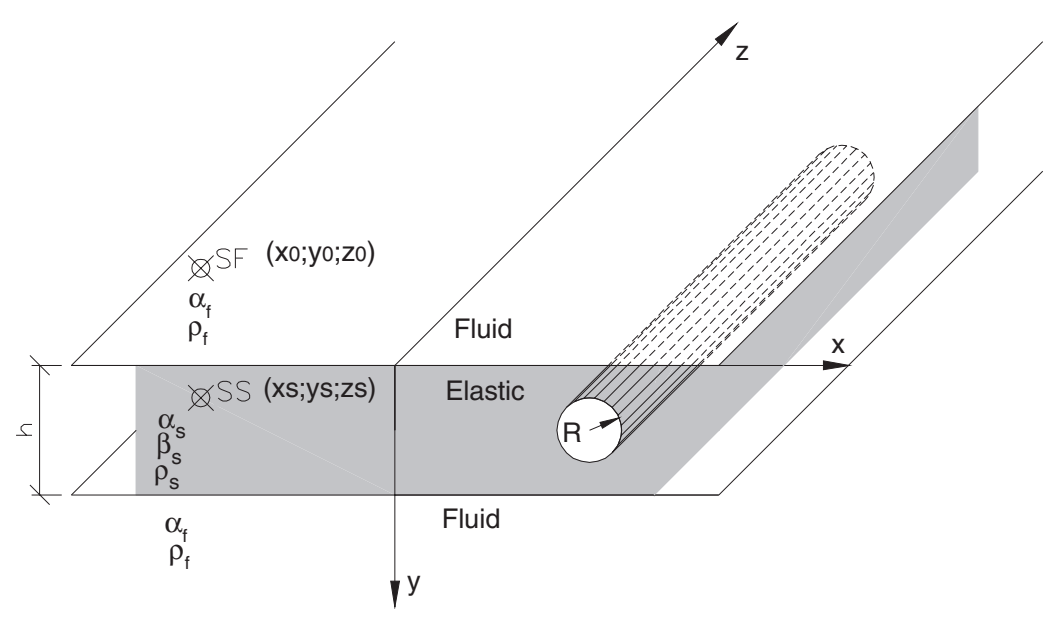

Fig. 1. Geometry of the problem.

modulus is computed as $\mu=\mu_{r}(1+\mathrm{i} \eta)$, where $\mu_{r}$ corresponds to the classic modulus and $\eta$ is the loss factor. The Lamé constant is written in the same form as the shear modulus.

In this paper the influence of a heterogeneity in the elastic partition on sound pressure level is studied by inserting a cylindrical circular inclusion with radius $R$, infinite along the $z$ direction, into the elastic layer. When the system is excited by a point load oscillating with a frequency $\omega$ and acting in the acoustic medium at $\left(x_{0}, y_{0}, z_{0}\right)$, the incident pressure field at a point $(x, y, z)$ can be obtained by the following expression:

$$
\sigma_{3 \mathrm{D}}^{\mathrm{full}}(\omega, x, y, z)=\frac{A \mathrm{e}^{\mathrm{i} \frac{\omega}{\alpha_{f}}\left(\alpha_{f} t-\sqrt{\left(x-x_{0}\right)^{2}+\left(y-y_{0}\right)^{2}+\left(z-z_{0}\right)^{2}}\right)}}{\sqrt{\left(x-x_{0}\right)^{2}+\left(y-y_{0}\right)^{2}+\left(z-z_{0}\right)^{2}}},
$$

in which $A$ is the wave amplitude and $\mathrm{i}=\sqrt{-1}$. As the geometry of the model is constant along the $z$ direction and the source is three-dimensional (3D), the incident field can also be obtained by calculating a series of 2D problems and applying a Fourier transformation in the $z$ direction. With this procedure, the responses are obtained in the frequency-wavenumber domain for varying effective wavenumbers, $k_{\alpha_{f}}=\sqrt{\left(\omega / \alpha_{f}\right)^{2}-k_{z}^{2}}$, with $\operatorname{Im}\left(k_{\alpha_{f}}\right) \leqslant 0$ and $k_{z}$ being the axial wavenumber. In this $k_{z}$ domain, the system is excited by spatially sinusoidal harmonic line loads acting at $\left(x_{0}, y_{0}\right)$ whose pressure field at a point $(x, y)$ is given by

$$
\sigma^{\text {full }}\left(\omega, x, y, k_{z}\right)=\frac{-\mathrm{i} A}{2} H_{0}^{(2)}\left(k_{\alpha_{f}} \sqrt{\left(x-x_{0}\right)^{2}+\left(y-y_{0}\right)^{2}}\right) \mathrm{e}^{-\mathrm{i} k_{z} z} .
$$

The former 3D pressure field is then calculated as a discrete summation of 2D problems obtained using expression (2), by applying an inverse Fourier transformation, and assuming the existence of an infinite number of sources placed along the $z$ direction at equal intervals, $L$. This equation converges and can be approximated by a finite sum of terms. The distance $L$ needs to be large enough to avoid spatial contamination.

The same procedure can be applied to point loads acting within the solid medium. Each 2D incident field produced by a spatially sinusoidal harmonic line load acting at a point $\left(x_{s}, y_{\mathrm{s}}\right)$ of the elastic medium can be expressed by the displacements $G_{i, j}$ full (where the index $i=x, y, z$ defines the direction in which the load is acting, while the second index, $j=x, y, z$, indicates the direction of the displacement) at a point $(x, y)$ according to the following expressions [10]:

$$
G_{i j}^{\text {full }}\left(\omega, x, y, k_{z}\right)=A\left[k_{s}^{2} H_{0 \beta}-\frac{1}{r} B_{1}+\gamma_{i}^{2} B_{2}\right], \quad \text { with } \quad i=j=x, y,
$$




$$
\begin{gathered}
G_{z z}^{\mathrm{full}}\left(\omega, x, y, k_{z}\right)=A\left[k_{s}^{2} H_{0 \beta}-k_{z}^{2} B_{0}\right], \\
G_{x y}^{\mathrm{full}}\left(\omega, x, y, k_{z}\right)=G_{y x}^{\mathrm{full}}\left(\omega, x, y, k_{z}\right)=\gamma_{x} \gamma_{y} A B_{2}, \\
G_{i z}^{\mathrm{full}}\left(\omega, x, y, k_{z}\right)=G_{z j}^{\mathrm{full}}\left(\omega, x, y, k_{z}\right)=\mathrm{i} k_{z} \gamma_{i} A B_{1} \quad \text { with } \quad i=j=x, y,
\end{gathered}
$$

where $A=1 / 4 \mathrm{i} \rho_{s} \omega^{2} ; \gamma_{x}=\left(x-x_{s}\right) / r ; \gamma_{y}=\left(y-y_{s}\right) / r ; B_{n}=k_{\beta}^{n} H_{n \beta}-k_{\alpha}^{n} H_{n \alpha} ; H_{n \alpha}=H_{n}^{(2)}\left(k_{\alpha_{s}} r\right)$ and $H_{n \beta}=$ $H_{n}^{(2)}\left(k_{\beta_{s}} r\right)$ are Hankel functions of the second kind and order $n ; k_{s}=\sqrt{\omega / \beta_{s}} ; r=\sqrt{\left(x-x_{s}\right)^{2}+\left(y-y_{s}\right)^{2}}$; $k_{\alpha_{s}}=\sqrt{\left(\omega / \alpha_{s}\right)^{2}-k_{z}^{2}}$ with $\operatorname{Im}\left(k_{\alpha_{s}}\right) \leqslant 0 ; k_{\beta_{s}}=\sqrt{\left(\omega / \beta_{s}\right)^{2}-k_{z}^{2}}$ with $\operatorname{Im}\left(k_{\beta_{s}}\right) \leqslant 0$ and $k_{z}=(2 \pi / L) n$.

In the same way, the scattered field produced by point loads with a heterogeneity inserted into the single layer can be evaluated by solving a sequence of $2 \mathrm{D}$ problems, with varying values of $k_{z}$.

\subsection{Green's functions for a single layered medium}

This section briefly describes the procedure used to obtain the $2.5 \mathrm{D}$ Green's functions for a single homogeneous elastic layer bounded by two fluid media, when excited by harmonic line loads with different $k_{z}$ values. These solutions have already been derived by Tadeu et al. [3] and can be expressed as the sum of the source terms equal to those in full space (which can be calculated according to above-defined expressions, (2) and (3)) and surface terms generated by the fluid/solid interfaces (interfaces $a$ and $b$, as in Fig. 2). The calculation of the surface terms requires knowledge of the solid layer displacement potentials and the pressure potentials generated by the solid/fluid surfaces. These potentials are written as a superposition of plane waves by means of a discrete wavenumber representation (after applying a Fourier transform in the $x$ direction). The integrals of the expressions are transformed into a summation by considering an infinite number of virtual plane sources distributed along the $x$ direction at equal intervals, $L_{x}$. In the fluid medium, pressure potentials

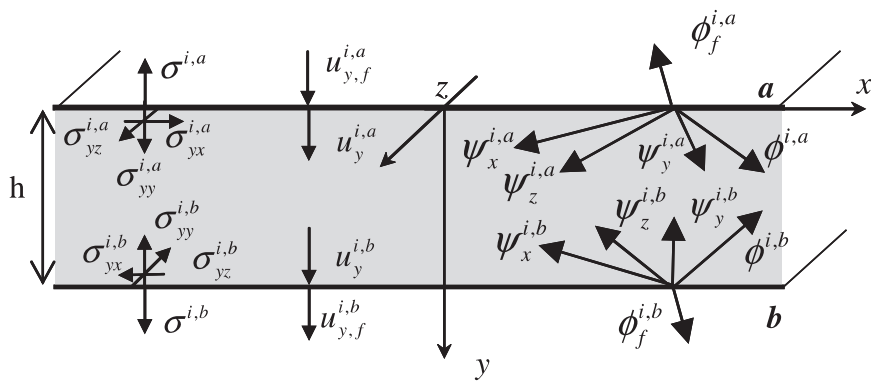

Fig. 2. Definition of potentials, stresses and displacements at the interfaces.

Table 1

Potentials generated at the interfaces when the load acts in the elastic medium along the $x$ direction

\begin{tabular}{llr}
\hline Interface $a$ & Interface $b$ \\
\hline Elastic medium & $\begin{array}{l}\phi^{x, a}=E_{a} \sum_{n=-N}^{n=+N}\left(\frac{k_{n}}{v_{n}} E_{b}^{a} A_{n}^{x}\right) E_{d} \\
\psi_{x}^{x, a}=0\end{array}$ & $\phi^{x, b}=E_{a} \sum_{n=-N}^{n=+N}\left(\frac{k_{n}}{v_{n}} E_{b}^{b} E_{n}^{x}\right) E_{d}$ \\
$\psi_{y}^{x, a}=E_{a} k_{z} \sum_{n=-N}^{n=+N}\left(\frac{E_{c}^{a}}{\gamma_{n}} B_{n}^{x}\right) E_{d}$ & $\psi_{x}^{x, b}=0$ & $\psi_{y}^{x, b}=E_{a} k_{z}^{n=+N} \sum_{n=-N}^{n}\left(\frac{E_{c}^{b}}{\gamma_{n}} F_{n}^{x}\right) E_{d}$ \\
& $\psi_{z}^{x, a}=-E_{a} \sum_{n=-N}^{n=+N}\left(E_{c}^{a} C_{n}^{x}\right) E_{d}$ & $\psi_{z}^{x, b}=E_{a}^{n=+N} \sum_{n=-N}^{n}\left(E_{c}^{b} G_{n}^{x}\right) E_{d}$ \\
Fluid medium & $\phi_{f}^{x, a}=-\frac{i}{L_{x}} \sum_{n=-N}^{n=+N}\left[\left(\frac{-\alpha_{f}^{2}}{\omega^{2} \lambda_{f}}\right) \frac{E_{f}^{a}}{v_{n}^{f}} D_{n}^{x}\right] E_{d}$ & $\phi_{f}^{x, b}=-\frac{i}{L_{x}} \sum_{n=-N}^{n=+N}\left[\left(\frac{-x_{f}^{2}}{\omega^{2} \lambda_{f}}\right) \frac{E_{f}^{b}}{v_{n}^{f}} H_{n}^{x}\right] E_{d}$ \\
\hline
\end{tabular}


Table 2

Potentials generated at the interfaces when the load acts in the elastic medium along the $y$ direction

\begin{tabular}{|c|c|c|c|}
\hline & Interface $a$ & & Interface $b$ \\
\hline Elastic medium & $\begin{array}{l}\phi^{y, a}=E_{a} \sum_{n=-N}^{n=+N}\left(E_{b}^{a} A_{n}^{y}\right) E_{d} \\
\psi_{x}^{y, a}=E_{a} k_{z} \sum_{n=-N}^{n=+N}\left(\frac{-E_{c}^{a}}{\gamma_{n}} C_{n}^{y}\right) E_{d} \\
\psi_{y}^{y, a}=0 \\
\psi_{z}^{y, a}=E_{a} \sum_{n=-N}^{n=+N}\left(\frac{k_{n}}{\gamma_{n}} E_{c}^{a} B_{n}^{y}\right) E_{d}\end{array}$ & & $\begin{array}{l}\phi^{y, b}=-E_{a} \sum_{n=-N}^{n=+N}\left(E_{b}^{b} E_{n}^{y}\right) E_{d} \\
\psi_{x}^{y, b}=E_{a} k_{z} \sum_{n=-N}^{n=+N}\left(\frac{-E_{c}^{b}}{\gamma_{n}} G_{n}^{y}\right) E_{d} \\
\psi_{y}^{y, b}=0 \\
\psi_{z}^{y, b}=E_{a} \sum_{n=-N}^{n=+N}\left(\frac{k_{n}}{\gamma_{n}} E_{c}^{b} F_{n}^{y}\right) E_{d}\end{array}$ \\
\hline Fluid medium & $\phi_{f}^{y, a}=-\frac{\mathrm{i}}{L_{x}} \sum_{n=-N}^{n=+N}\left[\left(\frac{-\alpha_{f}^{2}}{\omega^{2} \lambda_{f}}\right) \frac{E_{f}^{a}}{v_{n}^{f}} D_{n}^{y}\right.$ & $E_{d}$ & $\phi_{f}^{y, b}=-\frac{\mathrm{i}}{L_{x}} \sum_{n=-N}^{n=+N}\left[\left(\frac{-\alpha_{f}^{2}}{\omega^{2} \lambda_{f}}\right) \frac{E_{f}^{b}}{v_{n}^{f}} H_{n}^{y}\right]$ \\
\hline
\end{tabular}

Table 3

Potentials generated at the interfaces when the load acts in the elastic medium along the $z$ direction

\begin{tabular}{llr}
\hline Interface $a$ & Interface $b$ \\
\hline Elastic medium & $\phi^{z, a}=E_{a} k_{z} \sum_{n=-N}^{n=+N}\left(\frac{E_{b}^{a}}{v_{n}} A_{n}^{z}\right) E_{d}$ & $\phi^{z, b}=E_{a} k_{z} \sum_{n=-N}^{n=+N}\left(\frac{E_{b}^{b}}{v_{n}} E_{n}^{z}\right) E_{d}$ \\
$\psi_{x}^{z, a}=E_{a} \sum_{n=-N}^{n=+N}\left(E_{c}^{a} B_{n}^{z}\right) E_{d}$ & $\psi_{x}^{z, b}=-E_{a} \sum_{n=-N}^{n=+N}\left(E_{c}^{b} F_{n}^{z}\right) E_{d}$ \\
$\psi_{y}^{z, a}=E_{a} \sum_{n=-N}^{n=+N}\left(\frac{-k_{n}}{\gamma_{n}} E_{c}^{a} C_{n}^{z}\right) E_{d}$ & $\left.\psi_{y}^{z, b}=E_{a}^{n=+N} \sum_{n=-N}^{n=-k_{n}} E_{c}^{b} G_{n}^{z}\right) E_{d}$ & $\psi_{z}^{z, b}=0$ \\
$\psi_{z}^{z, a}=0$ & $\phi_{f}^{z, a}=-\frac{\mathrm{i}}{\nu_{x}} \sum_{n=-N}^{n=+N}\left[\left(\frac{-\alpha_{f}^{2}}{\omega^{2} \lambda_{f}}\right) \frac{E_{f}^{a}}{v_{n}^{f}} D_{n}^{z}\right] E_{d}$ & $\phi_{f}^{z, b}=-\frac{i}{L_{x}} \sum_{n=-N}^{n=+N}\left[\left(\frac{-x_{f}^{2}}{\omega^{2} \lambda_{f}}\right) \frac{E_{f}^{b}}{v_{n}^{f}} H_{n}^{z}\right] E_{d}$ \\
\hline Fluid medium &
\end{tabular}

Table 4

Potentials generated at the interfaces when the load acts in the fluid medium

\begin{tabular}{llr}
\hline Interface $a$ & Interface $b$ \\
\hline Elastic medium & $\phi^{f, a}=E_{a} \sum_{n=-N}^{n=+N}\left(E_{b}^{a} A_{n}^{f}\right) E_{d}$ & $\phi^{f, b}=-E_{a} \sum_{n=-N}^{n=+N}\left(E_{b}^{b} E_{n}^{f}\right) E_{d}$ \\
$\psi_{x}^{f, a}=E_{a} k_{z} \sum_{n=-N}^{n=+N}\left(\frac{-E_{c}^{a}}{\gamma_{n}} C_{n}^{f}\right) E_{d}$ & $\psi_{x}^{f, b}=E_{a} k_{z} \sum_{n=-N}^{n=+N}\left(\frac{-E_{c}^{b}}{\gamma_{n}} G_{n}^{f}\right) E_{d}$ \\
$\psi_{y}^{f, a}=0$ & $\psi_{y}^{f, b}=0$ & $\psi_{z}^{f, b}=E_{a} \sum_{n=-N}^{n=+N}\left(\frac{k_{n}}{\gamma_{n}} E_{c}^{b} F_{n}^{f}\right) E_{d}$ \\
$\psi_{z}^{f, a}=E_{a} \sum_{n=-N}^{n=+N}\left(\frac{k_{n}}{\gamma_{n}} E_{c}^{a} B_{n}^{f}\right) E_{d}$ & $\phi_{f}^{f, b}=-\frac{i}{L_{x}} \sum_{n=-N}^{n=+N}\left[\left(\frac{-x_{f}^{2}}{\omega^{2} \lambda_{f}}\right) \frac{E_{f}^{b}}{v_{n}^{f}} H_{n}^{f}\right] E_{d}$ \\
\hline Fluid medium & $\phi_{f}^{f, a}=-\frac{\mathrm{i}}{L_{x}} \sum_{n=-N}^{n=+N}\left[\left(\frac{-\alpha_{f}^{2}}{\omega^{2} \lambda_{f}}\right) \frac{E_{f}^{a}}{v_{n}^{f}} D_{n}^{f}\right] E_{d}$ & \\
\hline
\end{tabular}

$\left(\phi_{f}^{i, a}, \phi_{f}^{i, b}\right)$ are defined at the interfaces, whereas in the elastic medium, the wave field is expressed by means of pressure potentials $\left(\phi^{i, a}, \phi^{i, b}\right)$ and shear potentials $\left(\psi_{x}^{i, a}, \psi_{y}^{i, a}, \psi_{z}^{i, a}, \psi_{x}^{i, b}, \psi_{y}^{i, b}, \psi_{z}^{i, b}\right)$ with $i=x, y, z, f$ corresponding to the loads applied (as listed in Tables 1-4).

In the expressions listed in Tables $1-4$, the coefficients correspond to: $E_{f}^{a}=\mathrm{e}^{-\mathrm{i} r_{n}^{f|y|}} ; E_{f}^{b}=\mathrm{e}^{-\mathrm{i} v_{n}^{f|y-h|}} ; v_{n}^{f}=$ $\sqrt{k_{p_{f}}^{2}-k_{z}^{2}-k_{n}^{2}}$ with $\operatorname{Im}\left(v_{n}^{f}\right) \leqslant 0 ; E_{b}^{a}=\mathrm{e}^{-\mathrm{i} v_{n}^{|y|}} ; E_{b}^{b}=\mathrm{e}^{-\mathrm{i} v_{n}^{|y-h|}} ; E_{c}^{a}=\mathrm{e}^{-\mathrm{i} \gamma_{n}^{|y|}} ; E_{c}^{b}=\mathrm{e}^{-\mathrm{i} \gamma_{n}^{|y-h|}} ; \gamma_{n}=\sqrt{k_{s}^{2}-k_{z}^{2}-k_{n}^{2}}$ 
with $\operatorname{Im}\left(\gamma_{n}\right) \leqslant 0 ; v_{n}=\sqrt{k_{p}^{2}-k_{z}^{2}-k_{n}^{2}}$ with $\operatorname{Im}\left(v_{n}\right) \leqslant 0 ; E_{a}=1 / 2 \rho_{s} \omega^{2} L_{x} ; E_{d}=\mathrm{e}^{-\mathrm{i} k_{n}\left(x-x_{0}\right)} ; k_{n}=\left(2 \pi / L_{x}\right) n ; k_{p_{f}}=$ $\omega / \alpha_{f}$ and $k_{p}=\omega / \alpha_{s}$. The coefficients $A_{n}^{i}, B_{n}^{i}, C_{n}^{i}, D_{n}^{i}, E_{n}^{i}, F_{n}^{i}, G_{n}^{i}$, and $H_{n}^{i}$ with $i=x, y, z, f$ are unknowns which are determined by deriving the above-defined potentials in order to calculate stresses and displacements, and then establishing the appropriate boundary conditions: continuity of normal displacements $\left(u_{y}^{i, a}=u_{y, f}^{i, a}, u_{y}^{i, b}=u_{y, f}^{i, b}\right) \quad$ and stresses $\left(\sigma_{y y}^{i, a}=\sigma^{i, a}, \sigma_{y y}^{i, b}=\sigma^{i, b}\right)$ and null tangential stresses $\left(\sigma_{y x}^{i, a}=0, \sigma_{y z}^{i, a}=0, \sigma_{y x}^{i, b}=0, \sigma_{y z}^{l, b}=0\right)$ at the interfaces (see Fig. 2).

Once the unknown coefficients have been calculated, the displacements and stresses associated with the surface terms can be determined by applying partial derivatives to the potentials defined in Tables $1-4$. The Green's functions for the solid/fluid formation are then obtained from the sum of the source terms and the surface terms. When this has been done, expressions for displacements $G_{i, j}^{\text {surf }}$, in the elastic medium are given, as follows:

Load acting in the elastic medium in the $x$ direction:

$$
\begin{aligned}
G_{x x}^{\text {surf }} & =G_{x x}^{\text {full }}+E_{a} \sum_{n=-N}^{n=+N}\left[A_{n}^{x} \frac{-\mathrm{i} k_{n}^{2}}{v_{n}} E_{b}^{a}+\left(-\mathrm{i} \gamma_{n} C_{n}^{x}-\frac{\mathrm{i} k_{z}^{2}}{\gamma_{n}} B_{n}^{x}\right) E_{c}^{a}\right] E_{d}+E_{a} \sum_{n=-N}^{n=+N}\left[E_{n}^{x} \frac{-\mathrm{i} k_{n}^{2}}{v_{n}} E_{b}^{b}+\left(-\mathrm{i} \gamma_{n} G_{n}^{x}-\frac{\mathrm{i} k_{z}^{2}}{\gamma_{n}} F_{n}^{x}\right) E_{c}^{b}\right] E_{d}, \\
G_{x y}^{\text {surf }} & =G_{x y}^{\text {full }}+E_{a} \sum_{n=-N}^{n=+N}\left(-\mathrm{i} k_{n} A_{n}^{x} E_{b}^{a}+\mathrm{i} k_{n} C_{n}^{x} E_{c}^{a}\right) E_{d}+E_{a} \sum_{n=-N}^{n=+N}\left(\mathrm{i} k_{n} E_{n}^{x} E_{b}^{b}-\mathrm{i} k_{n} G_{n}^{x} E_{c}^{b}\right) E_{d} \\
G_{x z}^{\text {surf }} & =G_{x z}^{\text {full }}+E_{a} \sum_{n=-N}^{n=+N}\left(\frac{-\mathrm{i} k_{z} k_{n}}{v_{n}} A_{n}^{x} E_{b}^{a}+\frac{\mathrm{i} k_{z} k_{n}}{\gamma_{n}} B_{n}^{x} E_{c}^{a}\right) E_{d}+E_{a} \sum_{n=-N}^{n=+N}\left(\frac{-\mathrm{i} k_{z} k_{n}}{v_{n}} E_{n}^{x} E_{b}^{b}+\frac{\mathrm{i} k_{z} k_{n}}{\gamma_{n}} F_{n}^{x} E_{c}^{b}\right) E_{d}
\end{aligned}
$$

Load acting in the elastic medium in the $y$ direction:

$$
\begin{aligned}
G_{y x}^{\text {surf }} & =G_{y x}^{\mathrm{full}}+E_{a} \sum_{n=-N}^{n=+N}\left(-\mathrm{i} A_{n}^{y} k_{n} E_{b}^{a}+\mathrm{i} B_{n}^{y} k_{n} E_{c}^{a}\right) E_{d}+E_{a} \sum_{n=-N}^{n=+N}\left(-\mathrm{i} E_{n}^{y} k_{n} E_{b}^{b}+\mathrm{i} F_{n}^{y} k_{n} E_{c}^{b}\right) E_{d} \\
G_{y y}^{\text {surf }} & =G_{y y}^{\mathrm{full}}+E_{a} \sum_{n=-N}^{n=+N}\left[-\mathrm{i} v_{n} A_{n}^{y} E_{b}^{a}+\left(\frac{-\mathrm{i} k_{n}^{2}}{\gamma_{n}} B_{n}^{y}+\frac{-\mathrm{i} k_{z}^{2}}{\gamma_{n}} C_{n}^{y}\right) E_{c}^{a}\right] E_{d}+E_{a} \sum_{n=-N}^{n=+N}\left[-\mathrm{i} v_{n} E_{n}^{y} E_{b}^{b}+\left(\frac{-\mathrm{i} k_{n}^{2}}{\gamma_{n}} F_{n}^{y}+\frac{-\mathrm{i} k_{z}^{2}}{\gamma_{n}} G_{n}^{y}\right) E_{c}^{b}\right] E_{d}, \\
G_{y z}^{\text {surf }} & =G_{y z}^{\mathrm{full}}+E_{a} \sum_{n=-N}^{n=+N}\left(-\mathrm{i} A_{n}^{y} k_{z} E_{b}^{a}+\mathrm{i} C_{n}^{y} k_{z} E_{c}^{a}\right) E_{d}+E_{a} \sum_{n=-N}^{n=+N}\left(-\mathrm{i} E_{n}^{y} k_{z} E_{b}^{b}+\mathrm{i} G_{n}^{y} k_{z} E_{c}^{b}\right) E_{d}
\end{aligned}
$$

Load acting in the elastic medium in the $z$ direction:

$$
\begin{aligned}
G_{z x}^{\text {surf }} & =G_{z x}^{\text {full }}+E_{a} \sum_{n=-N}^{n=+N}\left(\frac{-\mathrm{i} k_{z} k_{n}}{v_{n}} A_{n}^{z} E_{b}^{a}+\frac{\mathrm{i} k_{z} k_{n}}{\gamma_{n}} C_{n}^{z} E_{c}^{a}\right) E_{d}+E_{a} \sum_{n=-N}^{n=+N}\left(\frac{-\mathrm{i} k_{z} k_{n}}{v_{n}} E_{n}^{z} E_{b}^{b}+\frac{\mathrm{i} k_{z} k_{n}}{\gamma_{n}} G_{n}^{z} E_{c}^{b}\right) E_{d}, \\
G_{z y}^{\text {surf }} & =G_{z y}^{\text {full }}+E_{a} \sum_{n=-N}^{n=+N}\left(-\mathrm{i} k_{z} A_{n}^{z} E_{b}^{a}+\mathrm{i} B_{n}^{z} k_{z} E_{c}^{a}\right) E_{d}+E_{a} \sum_{n=-N}^{n=+N}\left(-\mathrm{i} k_{z} E_{n}^{z} E_{b}^{b}+\mathrm{i} F_{n}^{z} k_{z} E_{c}^{b}\right) E_{d}, \\
G_{z z}^{\text {surf }} & =G_{z z}^{\text {full }}+E_{a} \sum_{n=-N}^{n=+N}\left[\frac{-\mathrm{i} k_{z}^{2}}{v_{n}} A_{n}^{z} E_{b}^{a}+\left(\frac{-\mathrm{i} k_{n}^{2}}{\gamma_{n}} C_{n}^{z}-\mathrm{i} \gamma_{n} B_{n}^{z}\right) E_{c}^{a}\right] E_{d}+E_{a} \sum_{n=-N}^{n=+N}\left[\frac{-\mathrm{i} k_{z}^{2}}{v_{n}} E_{n}^{z} E_{b}^{b}+\left(\frac{-\mathrm{i} k_{n}^{2}}{\gamma_{n}} G_{n}-\mathrm{i} \gamma_{n} F_{n}^{z}\right) E_{c}^{b}\right] E_{d} .
\end{aligned}
$$

Load acting in the fluid medium:

$$
\begin{aligned}
G_{f x}^{\mathrm{surf}} & =E_{a} \sum_{n=-N}^{n=+N}\left(-\mathrm{i} A_{n}^{f} k_{n} E_{b}^{a}+\mathrm{i} B_{n}^{f} k_{n} E_{c}^{a}\right) E_{d}+E_{a} \sum_{n=-N}^{n=+N}\left(-\mathrm{i} E_{n}^{f} k_{n} E_{b}^{b}+\mathrm{i} F_{n}^{f} k_{n} E_{c}^{b}\right) E_{d}, \\
G_{f y}^{\text {surf }} & =E_{a} \sum_{n=-N}^{n=+N}\left[-\mathrm{i} v_{n} A_{n}^{f} E_{b}^{a}+\left(\frac{-\mathrm{i} k_{n}^{2}}{\gamma_{n}} B_{n}^{f}+\frac{-\mathrm{i} k_{z}^{2}}{\gamma_{n}} C_{n}^{f}\right) E_{c}^{a}\right] E_{d}+E_{a} \sum_{n=-N}^{n=+N}\left[-\mathrm{i} v_{n} E_{n}^{f} E_{b}^{b}+\left(\frac{-\mathrm{i} k_{n}^{2}}{\gamma_{n}} F_{n}^{y}+\frac{-\mathrm{i} k_{z}^{2}}{\gamma_{n}} G_{n}^{f}\right) E_{c}^{b}\right] E_{d}, \\
G_{f z}^{\text {surf }} & =E_{a} \sum_{n=-N}^{n=+N}\left(-\mathrm{i} A_{n}^{f} k_{z} E_{b}^{a}+\mathrm{i} C_{n}^{f} k_{z} E_{c}^{a}\right) E_{d}+E_{a} \sum_{n=-N}^{n=+N}\left(-\mathrm{i} E_{n}^{f} k_{z} E_{b}^{b}+\mathrm{i} G_{n}^{f} k_{z} E_{c}^{b}\right) E_{d}
\end{aligned}
$$


The pressure $\sigma_{i}^{\text {surf }}$ with $i=x, y, z, f$ corresponding to the loads applied, in the fluid medium, can be obtained by applying the following expressions:

$$
\begin{gathered}
\sigma_{i}^{\text {surf }}=\kappa \sigma^{\text {full }}-\frac{\mathrm{i}}{L_{x}} \sum_{n=-N}^{n=+N}\left[\frac{E_{f}^{a}}{v_{n}^{f}} D_{n}^{i}\right] E_{d} \quad(\text { when } y<0), \\
\sigma_{i}^{\text {surf }}=-\frac{\mathrm{i}}{L_{x}} \sum_{n=-N}^{n=+N}\left[\frac{E_{f}^{b}}{v_{n}^{f}} H_{n}^{i}\right] E_{d} \quad(\text { when } y>h),
\end{gathered}
$$

where $\kappa=0$ if the source is in the elastic medium and $\kappa=1$ if the source is in the fluid.

The expressions for the Green's functions in the full medium $\sigma^{\text {full }}$ and $G_{i, j}^{\text {full }}$, can be defined in explicit form using expressions (2) and (3).

\subsection{BEM formulation}

Each 2D scattered field produced by the presence of a heterogeneity inside the layer is solved in the frequency domain by using the BEM. The model used in this work includes the above-defined 2.5D functions for a single-layer medium, thus only the boundaries of the heterogeneity need to be discretized. This may be rigid, free, fluid-filled or elastic-filled. If the inclusion is assumed to be elastic-filled, continuity of stresses and displacements are ascribed to the boundaries. By applying a set of three virtual loads to the outer part of the heterogeneity's boundary and another set of three virtual loads to the inner part of boundary, the full system of equations is obtained. Considering a virtual load acting at point $\underline{x}_{P}$ of the outer part of the boundary, in the $k$ direction, the boundary integral equations may be written as [11]:

$$
\begin{aligned}
& \sum_{l=1}^{3} \int_{S} t_{l}(\underline{x}, v, \omega) G_{k, l}^{\mathrm{surf}}\left(\underline{x}_{P}, \underline{x}, \omega\right) \mathrm{d} S+G_{k}^{\mathrm{inc}}\left(\underline{x}_{0}, \underline{x}_{P}, \omega\right) \\
& \quad=\sum_{l=1}^{3} \int_{S} u_{l}(\underline{x}, \omega) H_{k, l}^{\mathrm{surf}}\left(\underline{x}_{P}, \underline{x}, v, \omega\right) \mathrm{d} S+C_{k, l} u_{l}\left(\underline{x}_{P}, \omega\right),
\end{aligned}
$$

and if the load is applied at the inner boundary,

$$
\sum_{l=1}^{3} \int_{S} t_{l}(\underline{x}, v, \omega) G_{k, l}^{\mathrm{full}}\left(\underline{x}_{P}, \underline{x}, \omega\right) \mathrm{d} S=\sum_{l=1}^{3} \int_{S} u_{l}(\underline{x}, \omega) H_{k, l}^{\mathrm{full}}\left(\underline{x}_{P}, \underline{x}, v, \omega\right) \mathrm{d} s+C_{k, l} u_{l}\left(\underline{x}_{P}, \omega\right) .
$$

In these equations $u_{l}(\underline{x}, \omega)$ and $t_{l}(\underline{x}, v, \omega)$ describe displacements and stresses in direction $l$ at a point $\underline{x}$ of the boundary $S ; G_{k, l}^{\mathrm{surf}}\left(\underline{x}_{P}, \underline{x}, \omega\right)$ are the Green's functions for displacements in the single layered medium (obtained as described in the previous section) at point $\underline{x}$ in direction $l$ caused by a sinusoidal line load acting at the source point $\underline{x}_{P}$ in direction $k ; H_{k, l}^{\text {surf }}\left(\underline{x}_{P}, \underline{x}, v, \omega\right)$ are the Green's functions for traction components obtained by applying the well known equations relating strains and displacements; $H_{k, l}^{\text {full }}\left(\underline{x}_{P}, \underline{x}, v, \omega\right)$ and $G_{k, l}^{\text {full }}\left(\underline{x}_{P}, \underline{x}, \omega\right)$ are the Green's functions for traction and displacement components in a full space (full details of these functions can be found in Ref. [10]); $G_{k}^{\text {inc }}\left(\underline{x}_{0}, \underline{x}_{P}, \omega\right)$ is the incident displacement field when the source is placed at $\underline{x}_{0}$, obtained from the Green's functions described in the previous section; $v$ is the unit outward normal for boundary $S$; the subscripts $k, l=1,2,3$ denote the normal $(n)$, tangential $(t)$ directions relative to the boundary surface and $z$ directions; $C_{k, l}$ is a constant that equals $\delta_{k, l} / 2$ for a smooth boundary, where $\delta_{k, l}$ is the Kronecker delta function. Standard vector transformation operators are used to transform the Green's functions from the Cartesian coordinate system.

When the heterogeneity is assumed to be fluid-filled, Eq. (9) is simplified because only the continuity of displacements and normal stresses and null tangential stresses at the boundaries need to be verified. Eq. (10) becomes:

$$
\int_{S} p(\underline{x}, \omega) G_{f, l}^{\mathrm{full}}\left(\underline{x}_{P}, \underline{x}, \omega\right) \mathrm{d} S=\int_{S} u_{n}(\underline{x}, \omega) H_{f, l}^{\mathrm{full}}\left(\underline{x}_{P}, \underline{x}, \omega\right) \mathrm{d} S+C p\left(\underline{x}_{P}, \omega\right),
$$


where $p(\underline{x}, \omega)$ is the pressure at a point $\underline{x}$ of the boundary in the acoustic medium; $G_{f, l}^{\text {full }}\left(\underline{x}_{P}, \underline{x}, \omega\right)$ and $H_{f, l}^{\text {full }}\left(\underline{x}_{P}, \underline{x}, \omega\right)$ are the Green's tensors for normal displacement and pressure in a full fluid medium [10]; $C$ is a constant that equals $\frac{1}{2}$ for a smooth boundary and the subscript $l$ denotes here the normal direction to the boundary. If free or rigid inclusions are assumed only Eq. (9) is applied, simplified so that null stresses at the boundaries are confirmed for the former and null displacements for the latter.

The Boundary Integral equations are solved after discretization into $N$ constant boundary elements. The resulting integrations are calculated using a Gaussian quadrature scheme, except for the integrations of the Green's functions for the full space and the source terms of the Green's functions for the single solid layer, which are carried out analytically when the element to be integrated is the loaded element $[12,13]$.

Solving the resulting system makes it possible to obtain nodal solid displacements and stresses. The scattered wave field produced by the presence of the heterogeneity at any point of the domain can then be calculated by applying the Boundary Integral equation.

\section{Responses in the time domain}

The pressure field in the spatial-temporal domain is obtained by modelling a Ricker wavelet whose Fourier transform is

$$
U(\omega)=A\left[2 \pi^{1 / 2} t_{o} \mathrm{e}^{-\mathrm{i} \omega t_{s}}\right] \Omega^{2} \mathrm{e}^{-\Omega^{2}},
$$

in which $\Omega=\omega t_{o} / 2 ; A$ is the amplitude; $t_{s}$ is the time when the maximum occurs and $\pi t_{o}$ is the characteristic (dominant) period of the wavelet.

This wavelet form has been chosen because it decays rapidly, both in time and frequency, reducing computational effort and allowing easier interpretation of the computed time series and synthetic waveforms.

The Fourier transformations are obtained by discrete summations over wavenumbers and frequencies. Mathematically, this is achieved by adding periodic sources at spatial intervals $L_{x}=2 \pi / \Delta k_{n}$ (in the $x$-axis) with $\Delta k_{n}$ being the wavenumber step, and temporal intervals $T=2 \pi / \Delta \omega$ where $\Delta \omega$ is frequency step [14]. The spatial separation, $L_{x}$, must be large enough to guarantee that the response of the fictitious sources occurs at times later than $T$, thereby avoiding contamination. The analysis uses complex frequencies where $\omega_{c}=\omega-\mathrm{i} \zeta$, with $\zeta=0.7 \Delta \omega$, which further reduce the influence of the neighbouring fictitious sources and avoid the aliasing phenomena. In the time domain, this shift is later taken into account by applying an exponential window $\mathrm{e}^{\xi t}$ to the response [15].

\section{Verification of the model}

The BEM algorithm used in this work was implemented and verified by comparing the results with a BEM model where 2.5D Green functions for a full space are used. This model requires the discretization of the interfaces of both the layer and the heterogeneity. In order to limit the number of boundary elements used to discretize the interfaces of the layer, complex frequencies with an imaginary part are used $(\zeta=0.72 \pi / T)$. This considerably attenuates the contribution of the responses from the boundary elements placed at $L=2 \alpha_{s} T$, reducing the length of the interface to be discretized [15]. In our calculations a value of $T=0.02 \mathrm{~s}$ and $L=90 \mathrm{~m}$ were used to define this discretization.

Several verifications were performed, considering all the different types of inclusions. The responses provided by a cylindrical circular elastic inclusion $\left(\alpha_{s}=431.3 \mathrm{~m} \mathrm{~s}^{-1} ; \beta_{s}=282.9 \mathrm{~m} \mathrm{~s}^{-1} ; \rho_{s}=140.00 \mathrm{~kg} \mathrm{~m}^{-3}\right)$ with radius $R=3.0 \mathrm{~m}$, inside an elastic layer were chosen to illustrate the accuracy of the model (see Fig. 3(a)). The elastic layer $\left(\alpha_{s}=2182.2 \mathrm{~m} \mathrm{~s}^{-1} ; \beta_{s}=1336.3 \mathrm{~m} \mathrm{~s}^{-1} ; \rho_{s}=1400.00 \mathrm{~kg} \mathrm{~m}^{-3}\right)$ of thickness $h=15.0 \mathrm{~m}$ divides an infinite acoustic medium with a density $\rho_{f}=1000.00 \mathrm{~kg} \mathrm{~m}^{-3}$, allowing a dilatational wave velocity of $\alpha_{f}=1500.0 \mathrm{~m} \mathrm{~s}^{-1}$. The geometry was subjected to a dilatational harmonic line load applied at point $(0.0 \mathrm{~m}$; $-2.0 \mathrm{~m}$ ) of the fluid medium with $k_{z}=0.5 \mathrm{rad} \mathrm{m}^{-1}$. The responses were calculated at a receiver $\mathrm{R} 1$ placed in the medium containing the source $((4.0 \mathrm{~m} ;-1.5 \mathrm{~m}))$ and at a receiver $\mathrm{R} 2$ placed in the receiving fluid medium $((4.0 \mathrm{~m} ; 16.5 \mathrm{~m}))$. Computations are performed in the frequency range $2.0-256.0 \mathrm{~Hz}$, with a frequency step of $2.0 \mathrm{~Hz}$. The BEM model using Green's functions for a full space assumes surfaces discretized with 400 
(a)

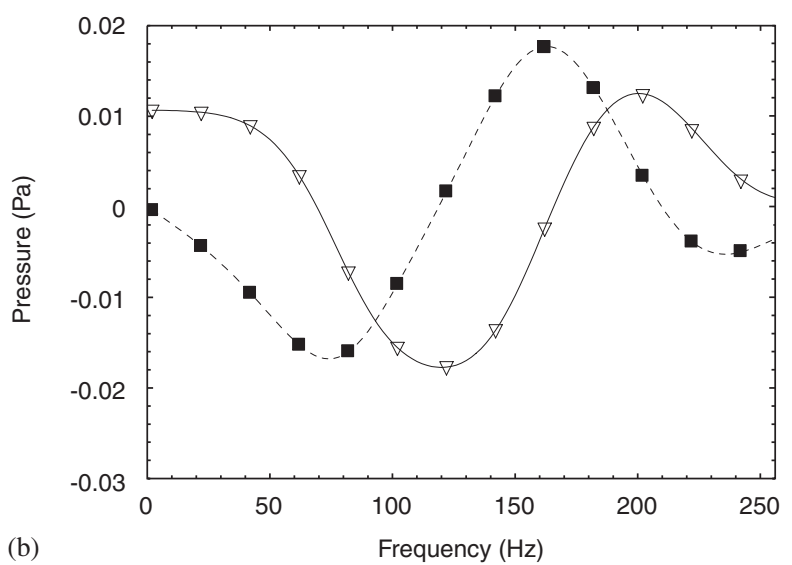

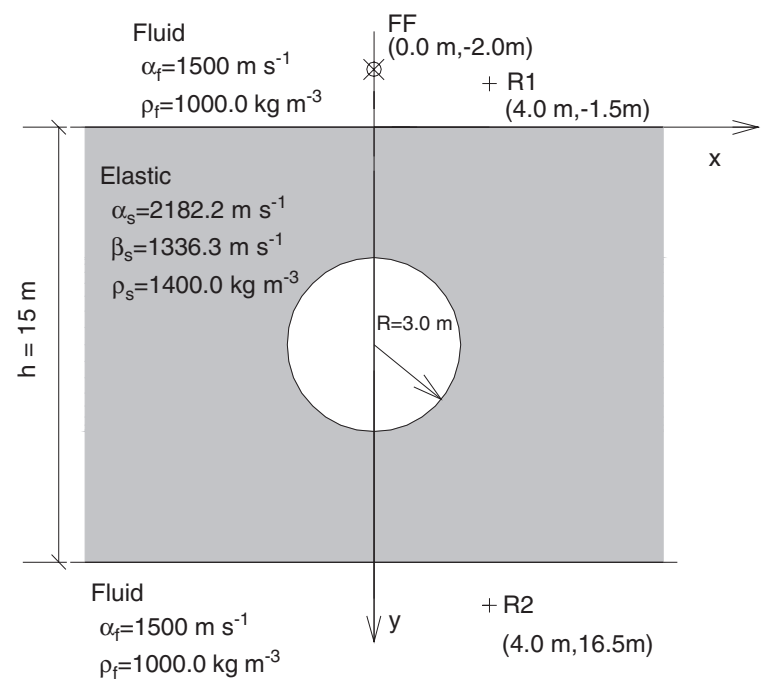

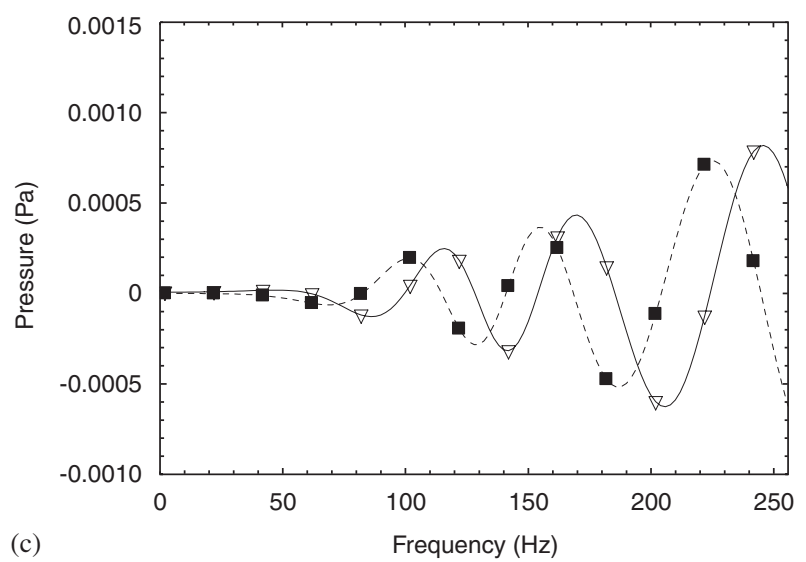

Fig. 3. Verification of the solution: (a) geometry; (b) pressure response at receiver R1; and (c) pressure response at receiver R2. ( - , Model 1: real part; ----, Model 1: imaginary part; $\nabla$, Model 2: real part; $\mathbf{\square}$, Model 2: imaginary part).

boundary elements and an inclusion modelled with 200 boundary elements. Using the BEM model described in this paper, only the heterogeneity is discretized, using 200 boundary elements. In order to illustrate the responses obtained in the verification, Figs. 3(b) and (c) display the scattered pressure recorded at receivers R1 and R2 obtained with the two models. In this figure, the solid line represents the solution obtained by the BEM model where all the interfaces are discretized (labelled in the plots as Model 1), and the marks illustrate the solution provided by the BEM model used in this work (labelled in the plots as Model 2). Analysis of the results confirms a good agreement between the two solutions. Equally good results were achieved for all the types of inclusions.

\section{Applications}

The contribution of a heterogeneity inside a single partition to the airborne sound insulation and impact sound pressure level is assessed. The responses provided by a homogeneous layer are used as a reference. Both frequency and time responses are computed. The partition is $0.20 \mathrm{~m}$ thick (see Fig. 4), infinite along its plane and divides an infinite acoustic medium. The layer is made of either concrete $\left(\alpha_{s}=3498.60 \mathrm{~m} \mathrm{~s}^{-1}\right.$; $\left.\beta_{s}=2245.00 \mathrm{~m} \mathrm{~s}^{-1} ; \quad \rho_{s}=2500.00 \mathrm{~kg} \mathrm{~m}^{-3} ; \quad \eta_{s}=6.00 \times 10^{-3}\right) \quad$ or ceramic material $\left(\alpha_{s}=2182.20 \mathrm{~m} \mathrm{~s}^{-1}\right.$; $\left.\beta_{s}=1336.30 \mathrm{~m} \mathrm{~s}^{-1} ; \rho_{s}=1400.00 \mathrm{~kg} \mathrm{~m}^{-3} ; \eta_{s}=1.50 \times 10^{-2}\right)$. The acoustic medium is air $\left(\rho_{f}=1.22 \mathrm{~kg} \mathrm{~m}^{-3}\right.$ and $\alpha_{f}=340.00 \mathrm{~m} \mathrm{~s}^{-1}$ ). 


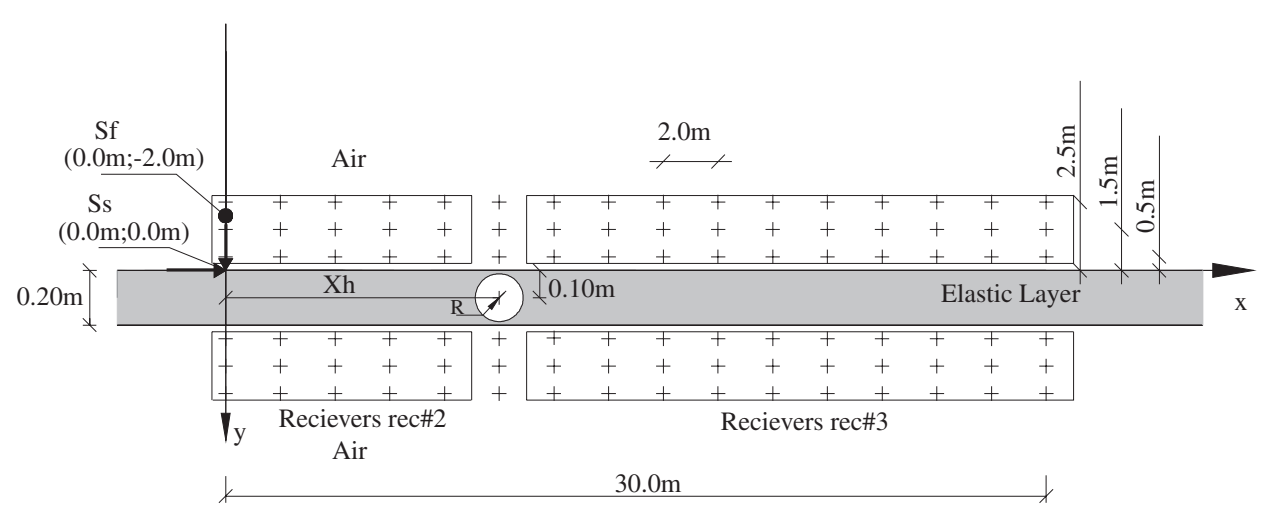

Fig. 4. Geometry of the model used in the simulations.

The model is excited by sources placed either in the acoustic medium $\left(S_{f}\right)$ at $(0.0 \mathrm{~m} ;-2.0 \mathrm{~m})$ or in the elastic medium $\left(S_{s}\right)$ at $(0.0 \mathrm{~m} ; 0.0 \mathrm{~m})$. The influence of the direction of the load in the elastic medium is studied by placing loads that can act either in the $y$ (vertical load) or $x$ (horizontal load) direction. Responses provided by rigid, free, fluid-filled and elastic-filled cylindrical circular inclusions are modelled. The fluid inside the fluidfilled heterogeneity may take the properties of air or water $\left(\rho_{f}=1000.00 \mathrm{~kg} \mathrm{~m}^{-3} ; \alpha_{f}=1500.00 \mathrm{~m} \mathrm{~s}^{-1}\right)$ and the elastic-filled heterogeneity is built with cork $\left(\alpha_{s}=431.30 \mathrm{~m} \mathrm{~s}^{-1} ; \beta_{s}=282.90 \mathrm{~m} \mathrm{~s}^{-1} ; \rho_{s}=140.00 \mathrm{~kg} \mathrm{~m}^{-3}\right.$; $\left.\eta_{s}=0.15\right)$.

The importance of the heterogeneity's size is addressed by modelling inclusions with different radii $(R=0.05,0.75$ and $0.09 \mathrm{~m})$. The influence of the inclusion's position is also considered by setting the centre of the heterogeneity at a depth of $0.10 \mathrm{~m}$ and changing its distance from the source along the $x$ direction, $x_{h}$ $\left(x_{h}=0.0,1.0,5.0\right.$ and $\left.10.0 \mathrm{~m}\right)$.

The frequency domain analysis uses a frequency range of $[2.0 ; 8192.0 \mathrm{~Hz}]$ with an increment of $2.0 \mathrm{~Hz}$. The responses are obtained by calculating the sound pressure over a grid of receivers equally spaced at distances of $2.0 \mathrm{~m}$ along the $x$ direction, as illustrated in Fig. 4.

Responses in the time domain are also computed using a total time given by $T=\frac{1}{2.0}=0.5 \mathrm{~s}$. The spatial distance between the virtual sources was set to $4 \alpha_{s} T$. The source used is a Ricker wavelet pulse with a characteristic frequency of $2000 \mathrm{~Hz}$.

The heterogeneity is modelled using constant boundary elements whose number varies with the excitation frequency. The minimum number of boundary elements used was always greater than 30 .

\subsection{Acoustic behaviour of a single partition with an air-filled heterogeneity}

Fig. 5 presents the frequency domain responses when the model is excited by a cylindrical line load that acts in the fluid medium. In this figure, the plots regard the responses for a single concrete layer without (Fig. 5(a)) and with (Fig. 5(b)) the presence of an air-filled heterogeneity with $R=0.075 \mathrm{~m}$ placed at $(10.0 \mathrm{~m} ; 0.10 \mathrm{~m})$. The responses were calculated at lines of receivers equally spaced at distances of $0.30 \mathrm{~m}$, placed $0.5 \mathrm{~m}$ from the layer's surfaces. Once the pressure responses generated by the pressure load and recorded by the two lines of receivers were computed, the airborne sound insulation was obtained by computing the differences between the sound pressure levels at receivers placed in the emitting and receiving space at the same $x$ location. In the Figures a greyscale is used to represent the airborne sound insulation amplitudes.

The analysis of Fig. 5(a) shows that airborne sound insulation increases as the frequency increases, particularly at receivers placed in the vicinity of the source. The coincidence effect (labelled ' $\mathrm{fc}$ ' in the plots) associated with the propagation of guided waves along the layer is visible. Notice that this effect only starts to be clearly visible at receivers placed further from the source. At higher frequencies the interactions between the incident and directly-reflected wave fields (taking place in the medium containing the source), occurring when the difference in the travel paths is a multiple of the wavelength, are quite noticeable (labelled 'fint' in the plots). When the layer contains a heterogeneity (see Fig. 5(b)), the resulting wave field is disturbed and 

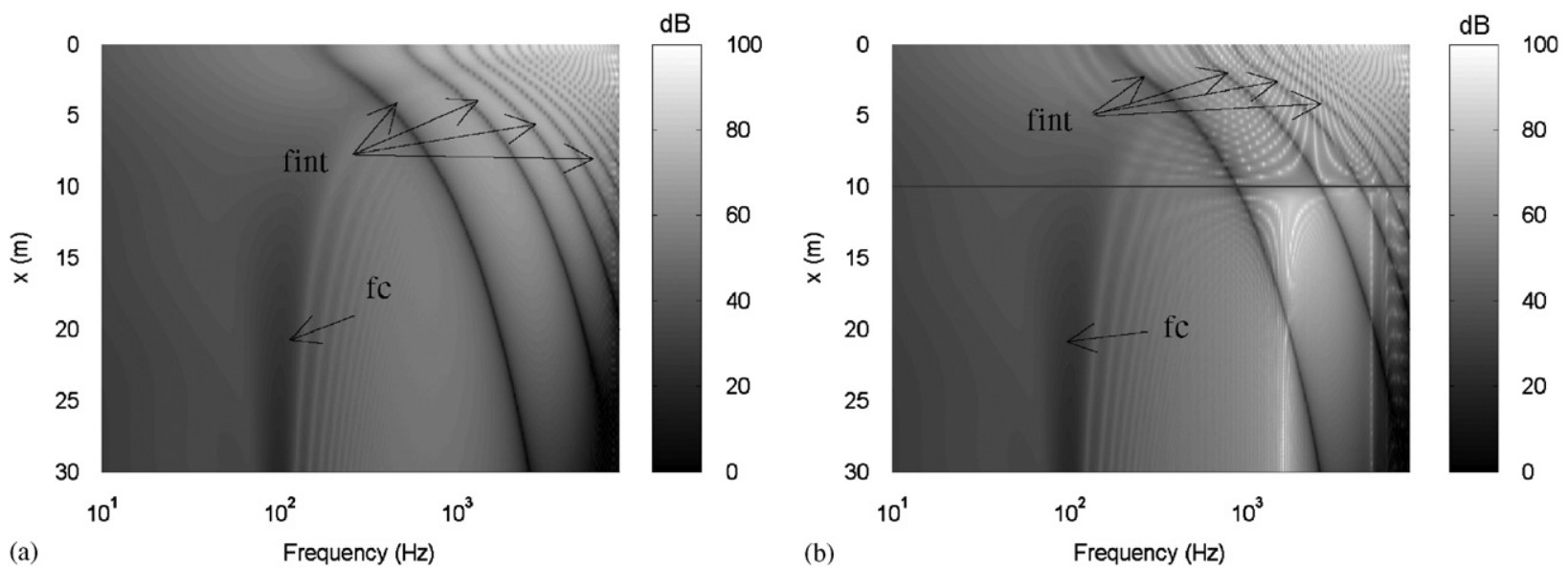

Fig. 5. Airborne sound insulation obtained at the line of receivers $0.5 \mathrm{~m}$ from the surface of a concrete layer: (a) homogeneous single layer and (b) single layer with an air-filled inclusion.
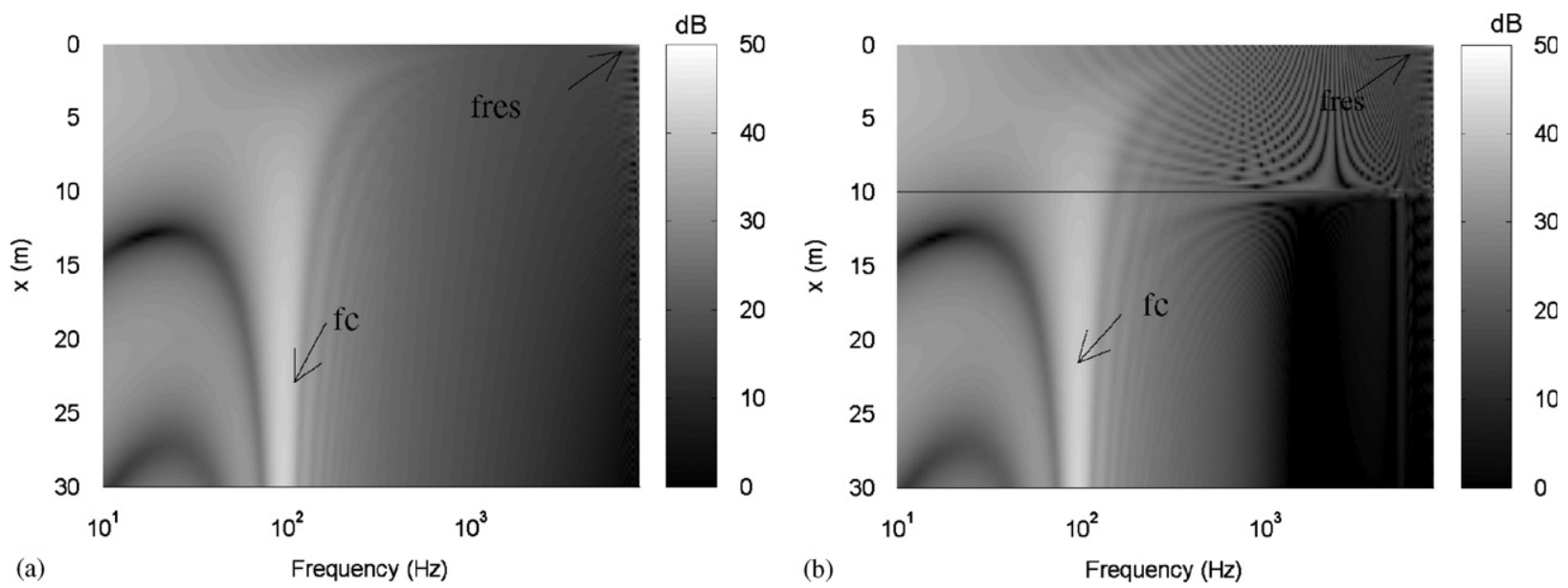

Fig. 6. Impact sound pressure level when the load acts in the $y$ direction (vertical) obtained at the line of receivers $0.5 \mathrm{~m}$ from the surfaces of a concrete layer: (a) homogeneous single layer and (b) single layer with an air-filled inclusion.

additional reflections occur. In this Figure a solid line represents the position of the inclusion. The responses provided by the single layer and by the same layer with the heterogeneity are very similar at low and medium frequencies (see Figs. 5(a) and (b)). At higher frequencies the airborne sound insulation is found to increase at receivers on the right of the inclusion. Moreover, at receivers on the left of the inclusion additional reflections produced by the inclusion occur, although not with a pronounced amplitude. At very high frequencies the airborne sound insulation again tends to approach that provided by the single layer. This phenomenon may be explained by waves travelling above and bellow the inclusion in the elastic layer.

The impact sound pressure level caused by the impact load acting in the vertical direction is also analysed for the single layer without (see Fig. 6(a)) and with the presence of the air-filled inclusion (see Fig. 6(b)). The responses were obtained by computing the sound pressure level in the acoustic receiving side.

When the load acts in the vertical direction, the impact sound level provided by the single homogeneous layer (see Fig. 6(a)) shows, as expected, a fall in sound level as frequency increases, except in the vicinity of the coincidence effect frequencies. The resonance effects occurring inside the elastic layer can be found at the end of the frequency domain response, at receivers placed near the load (labelled 'fres' in the plots). When the inclusion is inserted inside that layer (see Fig. 6(b)), the sound level at higher frequencies changes: the sound pressure level oscillates at receivers on the left of the inclusion while it decreases at receivers on the right of the 
inclusion, as a result of the wave field reflected by the inclusion. It can thus be concluded that the heterogeneity reduces the amount of waves propagating along the elastic layer, particularly at higher frequencies.

When the load excites the elastic medium in the $x$ direction the resulting responses show similar features to those registered when the load is applied in the $y$ direction (not illustrated). In this case the wavefield reflected on the inclusion appears to be stronger than that observed for the vertical load.

In order to better illustrate the physical phenomena described above, time domain responses, with the load in the acoustic medium, were computed for the set of receivers placed in the receiving medium, without (Fig. 7(a)) and with the air-filled inclusion (Fig. 7(b)).

Fig. 7(a) displays the responses obtained when the source is placed in the fluid medium in the presence of the homogeneous concrete layer. The plot registers a set of pulses that reach the receivers at different times. These are produced by refractions and mode conversions at the layer's surfaces. The first pulses recorded correspond to the refracted dilatational wave pulse (labelled as $P$ in the plot) and to the refracted shear elastic wave pulse (labelled as $S$ in the plot). A set of dispersive pulses then follows, generated in the guided waves that travel along the surfaces at different velocities. The last wave to arrive is the slowest one, the dilatational wave in the fluid (labelled in the plot as $P_{f}$ ). Notice that the pulses related to guided waves become more important at receivers placed at a certain distance from the source.

Fig. 7(b) presents the responses when the source $S_{f}$, excites the concrete layer with the air-filled heterogeneity. The plot shows that the receivers placed on the left of the inclusion (labelled in the plots as $x=0.0 \mathrm{~m} ; x=4.0 \mathrm{~m} ; x=8.0 \mathrm{~m}$ ) record another set of high frequency pulses (in relation to the single layer solution), appearing after the arrival of the refracted pulses produced by the layer. These refer to the reflected waves produced by the heterogeneity. The other receivers, on the right of the inclusion, show a fall in the amplitude of higher frequency pulses when compared with the solution provided by the homogeneous layer. Lower frequencies tend to travel along the layer without being affected by the presence of the inclusion. These features are in accordance with the frequency domain analysis.

Fig. 8(a) illustrates the time domain responses obtained when the source $S_{s}$, acting in the vertical direction, excites the homogeneous layer. The pulses recorded in this plot are related to the refractions occurring in the single layer medium followed by dispersive pulses due to propagation of the guided waves along the solid layer. When there is a heterogeneity (see Fig. 8(b)) extra pulses are added to the responses registered at the receivers on the left of the heterogeneity, referring to the reflected field produced by the inclusion. Moreover, the high frequency dispersive pulses recorded at the receivers on the right of the heterogeneity are attenuated.

Figs. 9(a) and (b) display the average airborne sound insulation. This is determined as follows: first, the average sound pressure level in each medium is computed as ten times the common logarithm of the ratio of the space average of the squared sound pressure to the square of the reference sound pressure; then the difference between the average of the sound pressure level in the incident and receiving media is plotted.

In these figures the average sound insulation curves provided by different sets of receivers are plotted. The sound insulation is determined from the average pressure at all receivers (henceforth referred to as rec $\# 1$ ); for
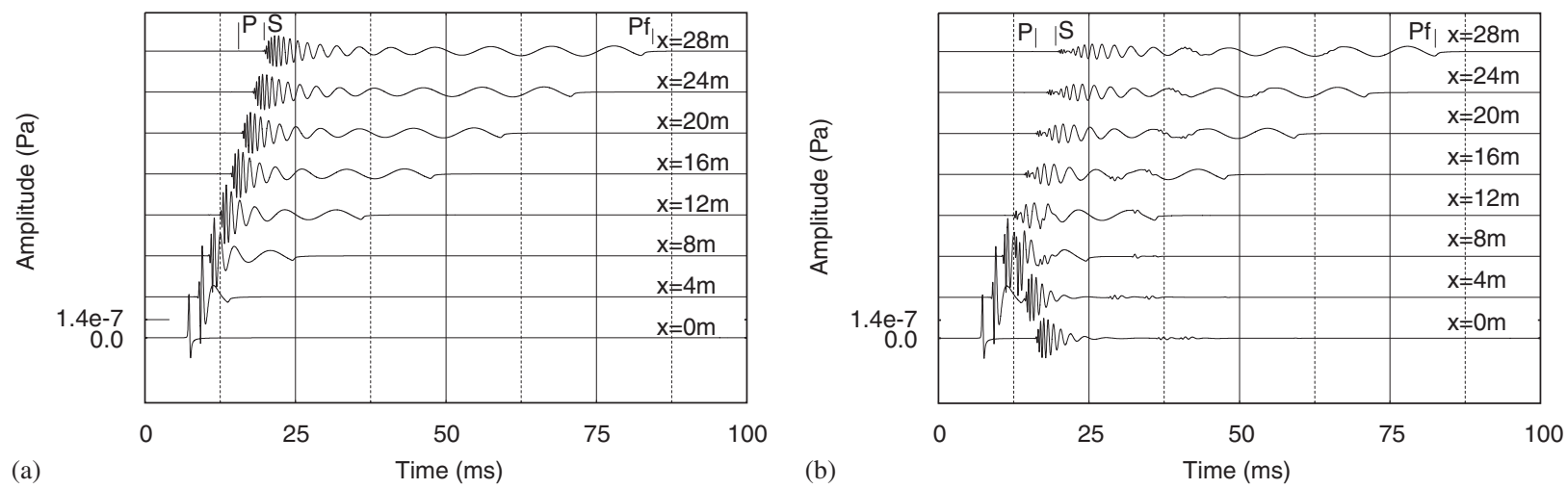

Fig. 7. Time domain pressure responses in the receiving medium produced by a source placed in the acoustic medium acting with a characteristic frequency of $2000 \mathrm{~Hz}$ : (a) homogeneous single layer and (b) single layer with an air-filled inclusion. 

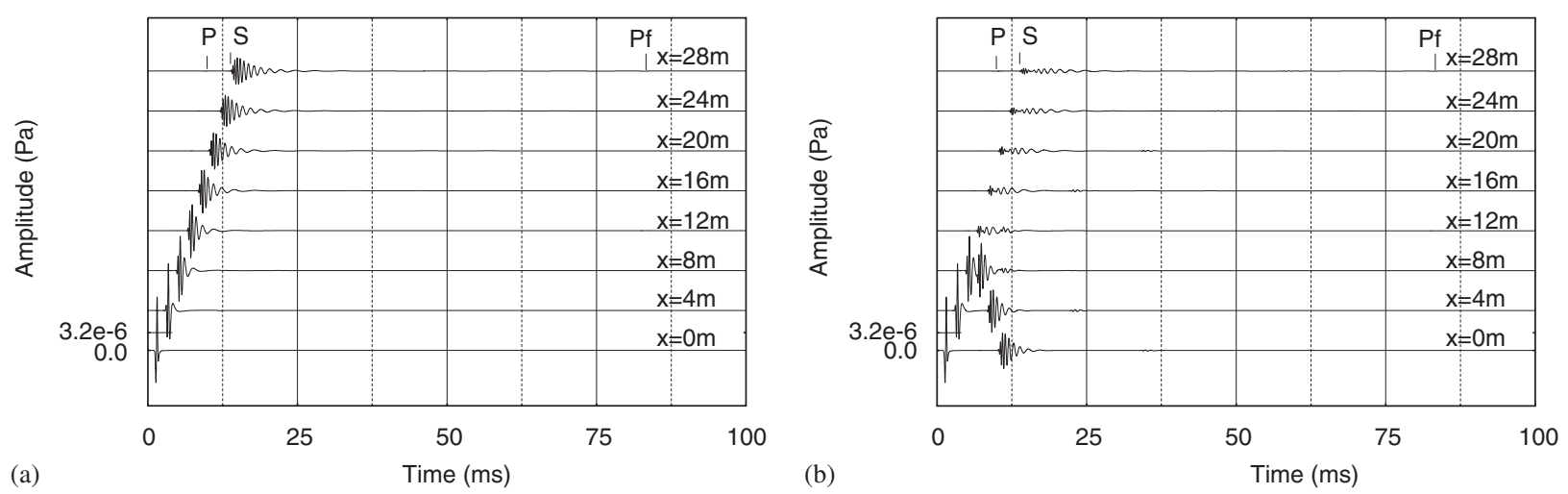

Fig. 8. Time domain pressure responses in the receiving medium produced by a source acting within the elastic medium in the $y$ direction (vertical) with a characteristic frequency of $2000 \mathrm{~Hz}$ : (a) homogeneous single layer and (b) single layer with an air-filled inclusion.
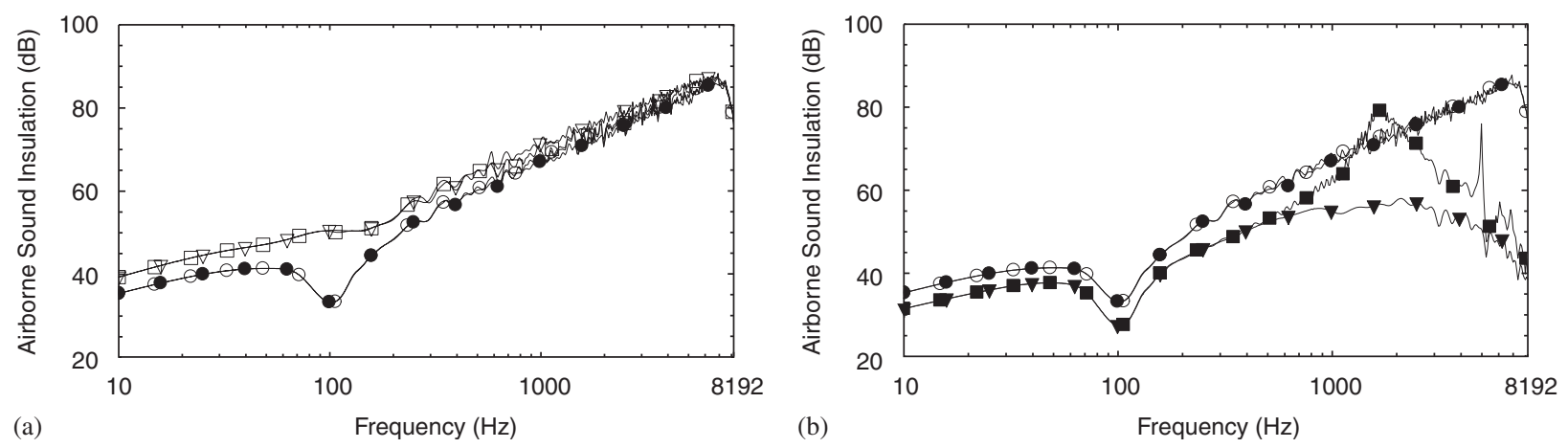

Fig. 9. Airborne sound insulation provided by a single concrete layer with an air-filled heterogeneity with radius $R=0.075 \mathrm{~m}$ placed at $x_{h}=10.0 \mathrm{~m}$ : (a) $r e c \sharp 1$ vs. $r e c \sharp 2$ and (b) $r e c \# 1$ vs. $r e c \# 3$ ( $\bullet$, homogeneous layer: $r e c \# 1 ; \nabla$, homogeneous layer: rec $\# 2$; 0 , air-filled heterogeneity: rec\#1; $\square$, air-filled heterogeneity: $r e c \sharp 2 ; \boldsymbol{\nabla}$, homogeneous layer: rec \#3; $\mathbf{\square}$, air-filled heterogeneity: rec\#3).

the receivers on the left of the inclusion (henceforth referred to as $r e c \sharp 2$ ), and from the responses at receivers on the right of the inclusion (henceforth referred to as $r e c \# 3$ ). In the figures the responses provided by the single layer are added for reference.

The airborne sound insulation provided by the homogeneous layer (see Fig. 9(a)) calculated considering all receivers $($ rec $\sharp 1)$ enables some of the acoustic phenomena above described to be observed (see comments to Fig. 5). A detailed analysis of this curve can be found in Ref. [4]. Comparisons of the curves provided by the single layers without and with the heterogeneity, show that, at higher frequencies, the airborne sound insulation is only slightly affected by the inclusion, and only small differences are found. In fact the airborne sound insulation calculated, taking the pressure at all receivers into account, does not allow a full description of the wave propagation features when the heterogeneity is present, as seen in Fig. 5. The average response is considerably influenced by the higher sound pressure levels that occur in the receiving medium at receivers placed closer to the source, given that the calculation is performed on an energy basis, enhancing the higher amplitudes. Thus, the lower amplitude signals recorded at receivers rec $\# 3$ are hidden in the results computed using all receivers. Therefore an analysis considering the average pressure recorded at receivers rec $\# 2$ and $r e c \# 3$ is performed to better quantify the influence of the heterogeneity. Anyway, for diagnostic purposes a uniformly spatial distribution of microphone positions should be used since the global results are more relevant.

Comparisons of the curves provided by the homogeneous layer (see Fig. 9(a)), obtained from receivers rec $\# 1$ and rec $\# 2$, shows that the airborne sound insulation indicated by receivers rec $\# 2$ is higher than that calculated using all receivers $($ rec $\# 1)$. This increase in insulation occurs mainly because the coincidence effect does not influence the sound level at receivers placed near the source. In fact, the propagation of the waves related to 
this effect is only captured at receivers placed further away from the source, therefore the critical frequency is not seen in the responses provided by rec $\# 2$ (see also comments in Figs. 5 and 7). Analysis of the curves indicated by receivers $r e c \# 2$, for the single partition without and with the heterogeneity, shows that the two curves are very similar. At higher frequencies small dips at specific frequencies are found (not clearly visible), produced by the layer containing the inclusion (related to the interaction between scattered pulses generated by the inclusion).

Analysis of airborne sound insulation provided by the homogeneous layer and captured at receivers rec $\# 1$ and rec\#3 (see Fig. 9(b)) shows that the response obtained by rec $\# 3$ is lower. In fact the incident field is lower at the receivers placed further away from the source owing to the attenuation of sound level due to the distance, thus the average airborne sound insulation using these receivers, decreases. Comparisons of the airborne sound insulation according to receivers $r e c \$ 3$ shows that there are major differences at higher frequencies between the solutions provided by the homogeneous layer and by the layer that contains a heterogeneity. These differences reveal an increase in the airborne sound insulation when the heterogeneity is present. At the end of the frequency domain response the sound insulation tends to approach that provided by the single layer.

When the load acts in the vertical direction (see Fig. 10(a)), the average impact sound level (obtained by averaging the sound pressure levels recorded at receivers placed in the receiving medium on an energy basis), calculated at all receivers ( $r e c \# 1)$ shows only small differences at higher frequencies when the layer contains an inclusion. The responses calculated for receivers $r e c \# 2$, again exhibit slightly higher sound pressure levels than those observed for the case of a homogeneous layer. Notice also that for this set of receivers the coincidence effect is not present. Analysis of the responses provided by receivers rec $\# 3$ (see Fig. 10(b)), shows that the heterogeneity reduces the propagation of waves along the elastic medium as the frequency increases.

\subsubsection{Influence of the position of the heterogeneity}

Fig. 11 presents the responses calculated at receivers $r e c \# 3$, assuming that the air-filled heterogeneity with radius $R=0.075 \mathrm{~m}$ is placed at positions $x_{h}=0.0,1.0,5.0$ and $10.0 \mathrm{~m}$. The airborne sound insulation provided by both the homogeneous layer and the same layer with a heterogeneity placed below the load $\left(x_{h}=0.0 \mathrm{~m}\right)$, are very similar. As the heterogeneity is placed further away from the position of the load, the airborne sound insulation increases for a larger frequency range at higher frequencies (see Fig. 11(a)).

When the inclusion is near the source more energy tends to be refracted to the elastic medium on the right of the heterogeneity, and thus more energy propagates along the layer. Therefore, the airborne sound insulation increases only slightly. If the inclusion is placed at a certain distance from the source, the wave field increases on the left of the inclusion, owing to scattered phenomena generated at the inclusion. Thus, less energy propagates along the layer to the right of the inclusion.
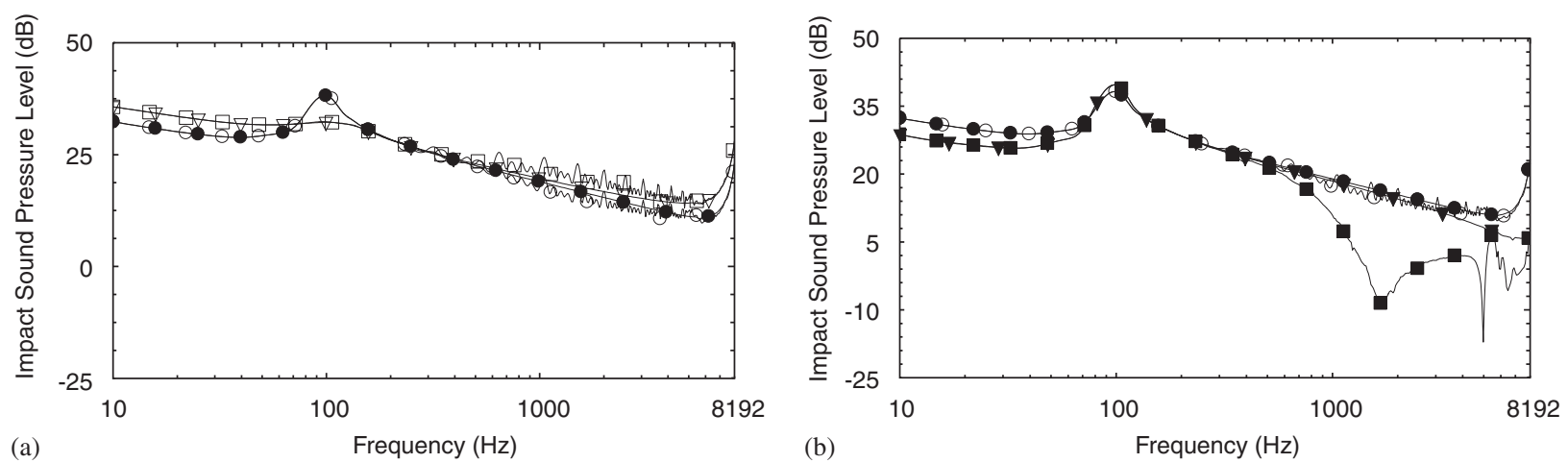

Fig. 10. Impact sound pressure level when the source acts in the $y$ direction (vertical) provided by a single concrete layer with an air-filled heterogeneity with radius $R=0.075 \mathrm{~m}$ placed at $x_{h}=10.0 \mathrm{~m}$ : (a) rec $\# 1$ vs. rec $\# 2$ and (b) rec $\# 1$ vs. rec $\# 3$ ( $\bullet$, homogeneous layer: rec $\# 1$; $\nabla$, homogeneous layer: $r e c \sharp 2 ; \bigcirc$, air-filled heterogeneity: $r e c \sharp 1 ; \square$, air-filled heterogeneity: rec $\sharp 2 ; \boldsymbol{\nabla}$, homogeneous layer: rec $\sharp 3$; $\mathbf{\square}$, air-filled heterogeneity: $\operatorname{rec} \sharp 3)$. 
The impact sound pressure level results (see Fig. 11(b)) show that the sound level increases at high frequencies, when the inclusion is placed below the heterogeneity. When the inclusion is placed at $x_{h}=1.0,5.0$ and $10.0 \mathrm{~m}$, the impact sound pressure levels provided by the partitions are similar and show a reduction in sound level at high frequencies.

\subsubsection{Influence of the size of the heterogeneity}

The influence of the size of the inclusion is assessed when the air-filled heterogeneity is placed inside the concrete partition at $x_{h}=10.0 \mathrm{~m}$ and its radius changes from $R=0.05,0.075$ to $0.09 \mathrm{~m}$ (see Fig. 12).

The responses computed at receivers rec $\# 3$ (see Fig. 12(a)) show that the airborne sound insulation increases as the size of the heterogeneity increases, at higher frequencies. This phenomenon starts to be observable at further lower frequencies as the size of the heterogeneity increases. Notice also that at the end of the response all plots tend to approach the single homogeneous layer solution. Impact sound pressure level (see Fig. 12(b)) falls, as expected, at similar frequency ranges to those observed in Fig. 12(a). Analysis of the responses provided by receivers $r e c \# 2$ lets us conclude that the scattered field produced by the heterogeneity on its left does not change significantly even when the inclusion is bigger (not displayed).

\subsubsection{Influence of the stiffness provided by the single layer}

The influence of the stiffness of the layer is assessed by changing the material of the partition from concrete to ceramic. Responses provided by receivers $r e c \sharp 3$ when the air-filled inclusion is placed at $x_{h}=10.0 \mathrm{~m}$, are plotted in Fig. 13. Analysis of these responses makes it possible to see that when the layer is made of ceramic,
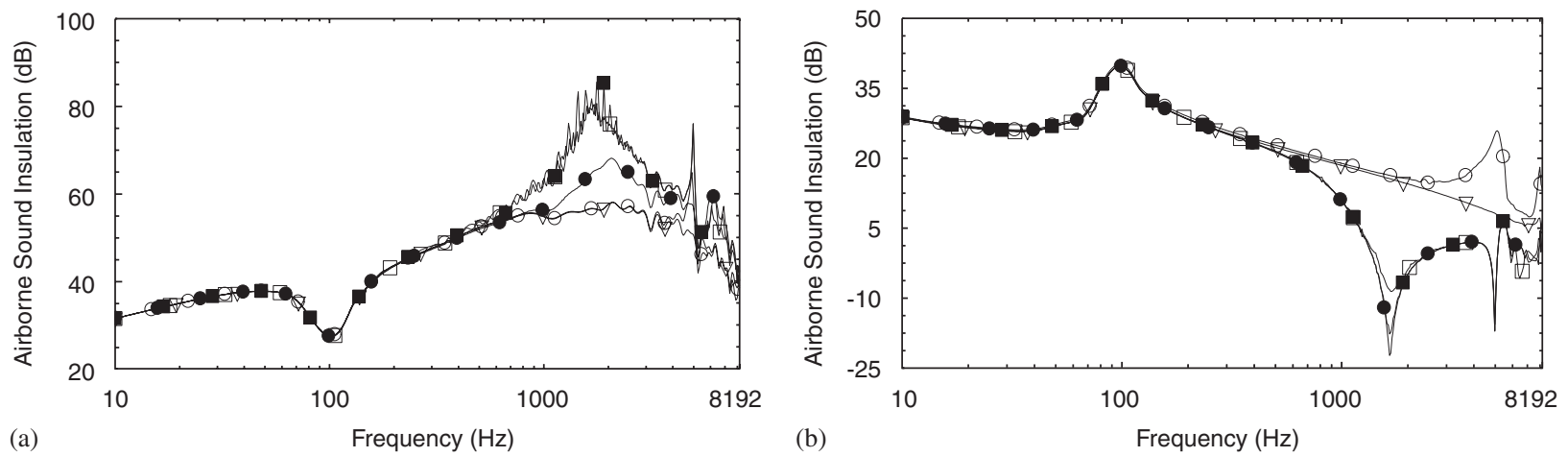

Fig. 11. Frequency domain responses provided by a single concrete layer with an air-filled inclusion with radius $R=0.075 \mathrm{~m}$ placed at different positions: (a) airborne sound insulation and (b) impact sound pressure level when the source acts in the $y$ direction (vertical) ( $\nabla$, homogeneous layer; $\bigcirc, x_{h}=0.0 \mathrm{~m} ; \boldsymbol{\bullet}, x_{h}=1.0 \mathrm{~m} ; \mathbf{\square}, x_{h}=5.0 \mathrm{~m} ; \square, x_{h}=10.0 \mathrm{~m}$ ).
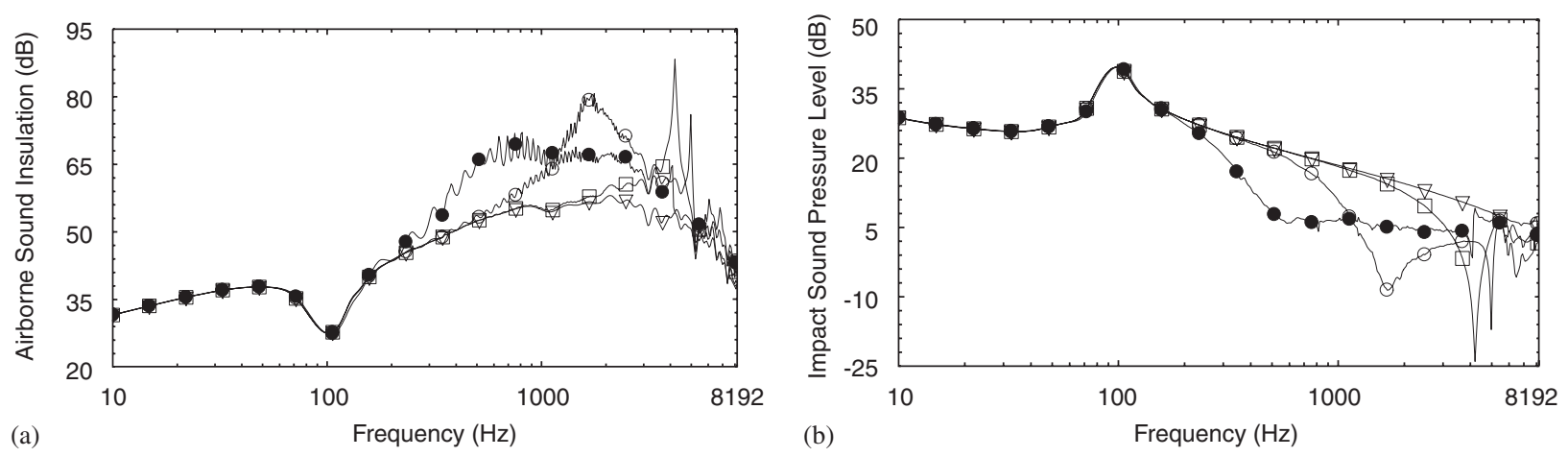

Fig. 12. Frequency domain responses provided by a single concrete layer with an air-filled inclusion for different radii: (a) airborne sound insulation and (b) impact sound pressure level when the source acts in the $y$ direction (vertical) ( $\nabla$, homogeneous layer; $\square, R=0.05 \mathrm{~m}$; $\bigcirc$, $R=0.075 \mathrm{~m} ; \boldsymbol{O}, R=0.09 \mathrm{~m})$. 

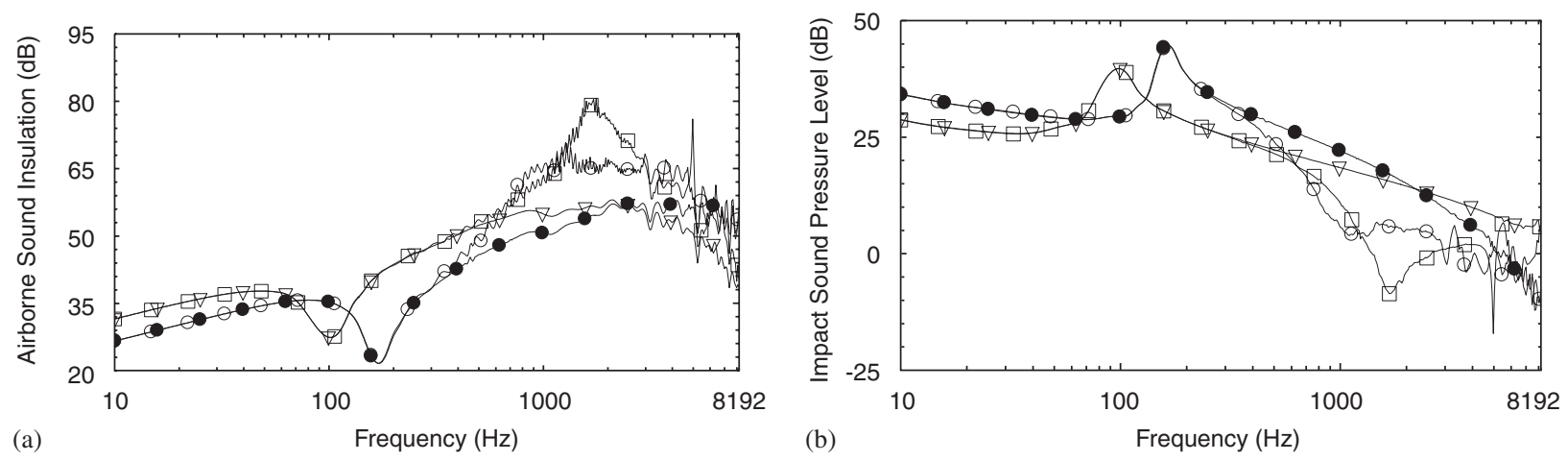

Fig. 13. Frequency domain responses provided by a single layer of either ceramic or concrete with an air-filled heterogeneity with radius $R=0.075 \mathrm{~m}$ placed at $x_{h}=10.0 \mathrm{~m}$ : (a) airborne sound insulation; and (b) impact sound pressure level when the source acts in the $y$ direction (vertical). ( $\bullet$, homogeneous ceramic layer; $\nabla$, homogeneous concrete layer; $\bigcirc$, ceramic layer with heterogeneity; $\square$, concrete layer with heterogeneity).
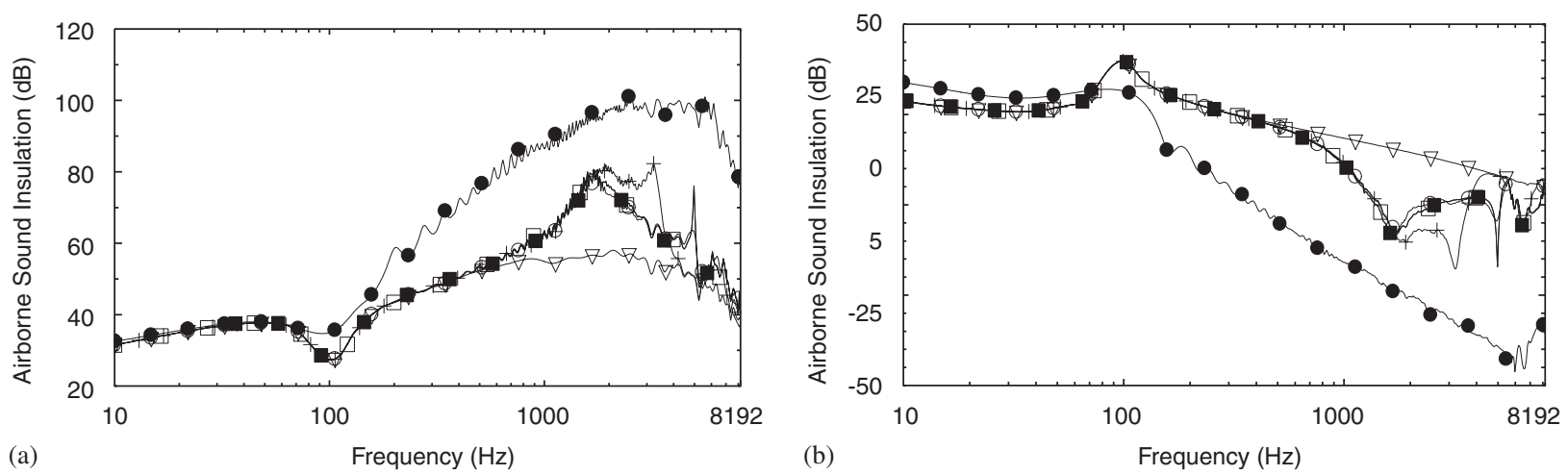

Fig. 14. Frequency domain responses provided by a single concrete layer with different types of heterogeneities with radius $R=0.075 \mathrm{~m}$ placed at $x_{h}=10.0 \mathrm{~m}$ : (a) airborne sound insulation and (b) impact sound pressure level when the source acts in the $y$ direction (vertical). ( $\nabla$, homogeneous layer; $\mathbf{\square}$, air-filled heterogeneity; + , water-filled heterogeneity; $\bigcirc$, cork-filled heterogeneity; $\bullet$, rigid heterogeneity; $\square$, free heterogeneity).

waves propagating at lower frequencies are more affected by the presence of the heterogeneity. As the panel's stiffness increases, both airborne sound insulation and impact sound pressure level responses are more affected in higher frequency domains.

\subsection{Acoustic behaviour of a single concrete partition with a free, a rigid, a fluid-filled and an elastic-filled heterogeneity}

In order to understand how the assumption of different boundary conditions at the heterogeneity's boundary may influence the responses, a rigid, a fluid (air or water filled) and an elastic (with the properties of cork) inclusion, with a radius $R=0.075 \mathrm{~m}$, placed inside the concrete layer at $x_{h}=10.0 \mathrm{~m}$, were modelled. Fig. 14 displays the computed responses using the records at receivers rec \#3.

The plots in Fig. 14 show that when a rigid heterogeneity is modelled the resulting airborne sound insulation is much higher than that produced by the homogeneous layer. On the other hand, the corresponding impact sound pressure level provided by the layer with the heterogeneity is lower than that given by the single layer. In fact, as the displacements in the boundaries of the inclusion are restricted, most of the energy that travels in the elastic medium hits the heterogeneity, and is reflected. The layer's movement is also reduced, and thus less energy propagates along it. For higher frequencies the waves propagate through the spaces between the inclusion and the surfaces more easily, causing a drop in airborne sound insulation and an increase in impact 
sound pressure level. When an elastic heterogeneity made of cork is assumed, the responses are very similar to those obtained for the free inclusion, and a clear attenuation is found in the wave propagation to the right of the inclusion. Cork is a flexible material and has the ability to dissipate energy, thus diminishing the wave propagation along the layer.

The results for an air-filled heterogeneity do not differ significantly in comparison with the ones obtained for the water-filled heterogeneity. In fact both tend to approach those provided by the cork-filled inclusion. Analysis of the responses computed using receivers $r e c \sharp 2$ shows that the curves provided by the free, fluidfilled and elastic-filled inclusions are quite similar (not displayed).

\section{Conclusions}

This work has described a BEM model developed in order to calculate the acoustic responses provided by a single layer with a heterogeneity. In this model only the boundaries of the inclusion were discretized since Green's functions are used for single layered medium bounded by acoustic media. The responses provoked by rigid, fluid-filled and elastic-filled inclusions were investigated. The model has been used to calculate pressure frequency and time responses due to cylindrical line loads acting in either the acoustic or the elastic medium. The results showed that at low and medium frequencies the responses provided by the single layer, with and without the presence of an air-filled inclusion, are very similar. At higher frequencies clear differences were found mainly at receivers placed on the right of the inclusion (opposite to the source). On the left of the inclusion only very small changes in the responses were identified. It was also found that the position and the size of the air-filled heterogeneity and the layer's material influence both the airborne sound insulation and impact sound pressure level responses on the right-hand side of the inclusion. The results provided by a corkfilled heterogeneity manifested a clear attenuation of wave propagation at medium and high frequencies.

\section{References}

[1] E.C. Sewell, Transmission of reverberant sound through a single leaf partition surrounded by an infinite rigid baffle, Journal of Sound and Vibration 12 (1970) 21-32.

[2] B.H. Sharp, Prediction methods for the sound transmission of building elements, Noise Control Engineering 11 (1978) $53-63$.

[3] A. Tadeu, J. António, 2.5D Green functions for elastodynamic problems in layered acoustic and elastic formations, Journal of Computer Modelling in Engineering and Sciences CMES (2002) 477-495.

[4] A. Tadeu, J. António, Acoustic insulation of single panel walls provided by analytical expressions versus the mass law, Journal of Sound and Vibration 257 (3) (2002) 457-475.

[5] L. Jong-Hwa, J. Ih, Significance of resonant sound transmission in finite single partitions, Applied Acoustics 277 (2004) $881-893$.

[6] J. Alba, J. Ramis, Modelling of impervious layers from measurements of sound reduction index, Applied Acoustics 64 (2003) 385-400.

[7] I.L. Vér, Relation between the normalized impact sound level and the sound transmission loss, The Journal of the Acoustical Society of America 50 (6) (1971) 1414-1417.

[8] E. Gerretsen, Calculation of sound transmission between dwellings by partitions and flanking structures, Applied Acoustics 12 (1979) 413-433.

[9] E. Gerretsen, Calculation of airborne and impact sound insulation between dwellings, Applied Acoustics 19 (1986) $245-264$.

[10] A. Tadeu, E. Kausel, Green's functions for two-and-a half dimensional elastodynamic problems, Journal of Engineering Mechanics ASCE 126 (10) (2000) 1093-1097.

[11] M.H. Aliabadi, The Boundary Element Method, Wiley, England, 2002.

[12] A. Tadeu, P. Santos, E. Kausel, Closed-form integration of singular terms for constant, linear and quadratic boundary elements. Part I: SH wave propagation, Engineering Analysis with Boundary Elements 23 (8) (1999) 671-681.

[13] A. Tadeu, P. Santos, E. Kausel, Closed-form integration of singular terms for constant, linear and quadratic boundary elements. Part II: SV-P wave propagation, Engineering Analysis with Boundary Elements 23 (9) (1999) 757-768.

[14] M. Bouchon, K. Aki, Discrete wave-number representation of seismic source wavefields, Bulletin of the Seismological Society of America 67 (1977) 259-277.

[15] E. Kausel, J.M. Roesset, Frequency domain analysis of undamped systems, Journal of Engineering Mechanics ASCE 118 (1992) 721-734. 